03

\title{
Теплопроводность за пределами закона Фурье
}

\author{
() А.И. Жмакин \\ Физико-технический институт им. А.Ф. Иофрфе РАН, \\ 194021 Санкт-Петербург, Россия \\ e-mail: a.zhmakin0@gmail.com
}

Поступило в Редакцию 18 июня 2020 г.

В окончательной редакции 18 июля 2020 г.

Принято к публикации 21 июля 2020 г.

Закон Фурье адекватно описывает теплоперенос в большинстве практических макроскопических задач. Однако в случаях теплообмена в быстропротекающих процессах, теплопереноса на микро- и наномасштабах, теплообмена в материалах с внутренней структурой (пористые среды, биологические ткани) требуются иные модели, учитывающие нелинейные эффекты, а также временную (память) и пространственную нелокальность. Рассмотрены такие модели, включая модели с задержкой, фононные и термодинамические модели, а также модели, использующие дифференциальные уравнения в дробных производных.

Ключевые слова: закон Фурье, DPL-модель, термоперенос, термодинамические и дробные модели, релаксон.

DOI: $10.21883 /$ JTF.2021.01.50267.207-20

\section{Введение}

Модель Фурье основана на определяющем соотношении

$$
\mathbf{q}(\mathbf{r}, t)=-\lambda \nabla T(\mathbf{r}, t),
$$

которое после подстановки в уравнение сохранения энергии для покоющегося твердого тела

$$
\frac{\partial(\rho C T)}{\partial t}=\nabla \mathbf{q}+Q
$$

приводит к классическому параболическому уравнению теплопроводности (также называемому уравнением Фурье-Киркгофа)

$$
\frac{\partial(\rho c T)}{\partial t}=\nabla \cdot(\lambda \nabla T)+Q
$$

Закон Фурье может быть непосредственно выведен в рамках классической неравновесной термодинамики, основанной на гипотезе локального равновесия [1].

С точки зрения неравновесной термодинамики закон Фурье описывает линейную связь между обобщенной силой (градиент температуры) и обобщенным потоком (тепловой поток) [2].

Закон Фурье справедлив, если

$$
\begin{array}{ll}
\text { - } & \frac{L}{\Lambda} \gg \mathrm{O}(1), \\
\text { - } & \frac{t}{\tau} \gg \mathrm{O}(1), \\
\text { - } & T \gg 0 \mathrm{~K},
\end{array}
$$

где $L$ - характерный размер системы, $\Lambda$ - средняя длина свободного пробега носителей тепла, $\tau$ - время релаксации. Отношение $\mathrm{Kn}=\Lambda / L$ называется числом Кнудсена как в динамике разреженных газов.

Тепловые волны в виде „второго звука“ [3] в гелии II при $1.4 \mathrm{~K}$ наблюдались В. Пешковым в 1944 г. со скоростью около $19 \mathrm{~m} / \mathrm{s}$, что на порядок величины меньше скорости звука в гелии II [4].

Позднее второй звук наблюдался при криогенных условиях в других материалах [5,6] твердом гелии-3 [7], флуориде натрия при $10 \mathrm{~K}$ [8-10]), висмуте (при $3.4 \mathrm{~K}[11])$, сапфире, титанате стронция [12], в графите при температуре выше $100 \mathrm{~K}$ [13].

Волновая природа распространения тепла и релаксационные процессы становятся доминирующими, а эффекты „памяти“ материала и нелинейные и нелокальные эффекты существенными при:

- ультрабыстром нагреве (лазерный нагрев и плавление $[14]^{1}$, например, фемтосекундном нагреве металлических пленок [16-18] или тонких пленок твердого аргона [19]), быстром затвердении жидких металлов [20], переходе в стеклообразное состояние переохлажденных жидкостей [21], экспериментах с импульсами тепла при комнатной температуре [22];

- теплопереносе на наномасштабах [23-26] (микроэлектронные приборы [27-30], например, „горячие“ в нанотранзисторах [31-34], наноструктурные термоэлектрические приборы [35], гетероструктуры [36], лазерная плазма при облучении маленьких мишеней [37]), теплообмен в ДНК при денатурации („плавлении“) развертывании двойной спирали в две обособленные полосы $[38]^{2}$;

\footnotetext{
${ }^{1}$ Импульсный лазер обеспечивает лучшую локализацию тепла по сравнению с непрерывным лазером [15].

2 Закон Фурье значительно переоценивает скорость диссипации тепла от источников с размерами меньше длины свободного пробега фононов [39], что существенно при анализе тепловых режимов микроэлектронных приборов. Эта проблема, однако, становится не столь острой с учетом нового явления „коллективной диффузии“,
} 
- теплопереносе в гранулированных и пористых средах $[40,41]$, включая пористый кремний [42];

- теплопереносе в биологических тканях [43-49].

Следует заметить, что ошибки, связанные с использованием закона Фурье вне области его применимости, иногда несущественны. Например, Вилсон и Кахилл [50] перечислили причины, по которым ошибки в определении теплопроводности алмаза не важны при анализе тепловых режимов микроэлектронных приборов, использующих алмазные распределители тепла:

1) баллистико-диффузионные эффекты в поликристаллических пленках алмаза, выращенных методом химического осаждения из газовой фазы, значительно меньше, чем в монокристаллах из-за рассеяния фононов на границах зерен;

2) по крайней мере, в GaN-транзисторах с высокой подвижностью электронов теплопроводность подложки существенна только при размерах активной области, испытывающей перегрев, более $1 \mu \mathrm{m}$;

3) при достаточно высокой плотности приборов горизонтальные градиенты температуры малы.

Импульсные лазеры с длительностью импульса от наносекунд до фемтосекунд используются в широком спектре медицинских технологий: оптическая томография [51], фотодинамическая терапия [15,52], гипертермия [53-57]. Контроль температуры в тканях может быть усилен путем инъекции наноструктур [58].

Источник возникновения запаздывания по времени, присутствие в материале разных носителей энергии [59] (хорошо известный в физике твердого тела пример релаксация энергии между электронной и фононной подсистемами), например, перенос энергии от свободных электронов решетке $[60,61]$ в металлах, нагреваемых ультракороткими лазерными импульсами $[62,63])$, или в материалах с гетерогенной внутренней структурой.

Биологические ткани содержат клетки, суперструктуры, жидкие и твердые элементы. Нагрев или охлаждение биологических тканей вызывает серию химических, электрических и механических процессов, например, диффузию, изменение электрического потенциала, осмотические потоки через клеточную мембрану; клеточные мембраны могут запасать энергию [64]. Таким образом, распространение тепла в биологических тканях задействует энергетические обмены на разных уровнях [64-66].

Известны различные подходы к экспериментальному исследованию теплопереноса на микро- и наномасштабах:

- $3 \omega$-метод [67] (этот метод основан на измерении третьей гармоники в напряжении при нагреве образца синусоидальной волной с частотой $\omega)$;

- сканирующая тепловая микроскопия [68];

открытого недавно Хугенбум-Потом и др. [39]: когда расстояние между наноразмерными источниками тепла становится малым в сравнении с длиной свободного пробега фононов, возникает рассеяние фононов на фононах, „происходящих“ из соседнего источника тепла, что увеличивает интенсивность диссипации тепла.
- биметаллические приборы;

- оптические методы;

- когерентные рентгеновские методы;

- исследования тепловых отражений.

В последнее время активно используются вычислительные методы:

- вычисления, основанные на „первых принципах“ (ab initio);

- неравновесная функция Грина;

- метод молекулярной динамики [69];

- метод Монте-Карло;

- многомасштабные вычисления.

В однородных материалах время релаксации составляет от $10^{-8}$ до $10^{-14} \mathrm{~s}$ [[70-72] (например, это время равно 3 ps для кремния [73], 4.-6.4 ps для ртути, $5.1-7.3$ ps для расплавленного галлия [74]), однако время релаксации в гранулированных средах и биологических объектах может составлять до 30 s. Например, Камински [70] сообщил о релаксационном времени $20 \mathrm{~s}$ для песка и $30 \mathrm{~s}$ для $\mathrm{NaHCO}_{3}$, Митра и др. [75] измерили релаксационное время в $15 \mathrm{~s}$ для обработанного мяса.

Однако Грассман и Петерс [40] и Хервиг и Бекерт [76] не обнаружили доказательств гиперболического характера распространения тепла в материалах с неоднородной внутренней структурой. Эти расхождения Ретцел и др. [77] объяснили методическими погрешностями ранних экспериментов независимым определением теплофизических свойств материала и измерением релаксационного времени. Ретцел и др. все параметры находили одновременно из единого эксперимента. Они подтвердили отклонения от закона Фурье, но получили меньшие значения релаксационного времени (2.26 s для песка, $1.77 \mathrm{~s}$ для обработанного мяса).

В более поздних экспериментах Антаки и др. [78] измерили релаксационное время $2 \mathrm{~s}$ для обработанного мяса.

О наблюдениях тепловых волн в живых тканях см. обзор [79]. Ошибки в предсказании распределения температуры в тканях при криохирургии и криосохранении могут проявляться в виде термоупругих напряжений [80-82] и появлении трещин в тканях $[83,84]$ изза значительного теплового расширения [85]. Механические волны наблюдались также в пленках твердого аргона при внезапном нагреве [86].

Yu T.Н. и др. [87] использовали метод низкочастотного импеданса для изучения реакции биологических тканей на мгновенное переключение сильного охлаждения и нагрева.

Для анализа теплопереноса в живых биологических тканях необходимо учесть вклад в теплообмен потоков артериальной и венозной крови $[49,88,89]$.

Континуальные модели теплопереноса с учетом кровеносной системы разрабатываются путем осреднения эффекта большого числа кровеносных сосудов в рассматриваемой области биологических тканей. Наиболее известная и, безусловно, самая важная континуальная 
модель была предложена в 1948 г. Пеннесом [90] и называется уравнением Пеннеса (иногда также „моделью теплового стока“ [91])

$$
\begin{gathered}
\rho c \frac{\partial T}{\partial t}=\nabla \cdot \lambda \nabla T+Q_{p}, \\
Q_{p}=c_{b} \omega_{b}\left(T_{a}-T\right)+\dot{q}_{m e t}+Q_{e x t},
\end{gathered}
$$

где $T, \rho, c$ и $\lambda$ - температура, плотность, удельная теплоемкость и теплопроводность тканей как однородной среды, $\omega_{b}-$ скорость перфузии крови, $c_{b}-$ удельная теплоемкость крови, $T_{a}$ - температура артериальной крови, $\dot{q}_{m e t}$ и $Q_{e x t}-$ тепловые источники изза метаболических реакций (этими источниками обычно можно пренебречь в задачах криобиологии) и внешний источник энергии.

Очевидное расширение, приводящее к нелинейному („модифицированному“) уравнению Пеннеса, состоит в учете температурной зависимости скорости перфузии крови $\omega_{b}=\omega_{b}^{0}+\omega_{b}^{1} T[92]$.

Отклонения от закона Фурье наблюдаются также для объектов пониженной размерности (систем, ограниченных в пространстве [93]) таких, как тонкие пленки, углеродные и нитрид-боровые нанотрубки, нанопроволоки, полосы графена [94-96], полимерные цепи [97,98]. Объекты низкой размерности демонстрируют так называемый „эффект масштаба“ или „размерный эффект“: теплопроводность уменьшается с размером образца. Например, теплопроводность кристаллических нанопроволок значительно ниже значений для объемного материала и уменьшается с диаметром проволоки [42] и увеличением шероховатости поверхности [99].

Объясняющая такое поведение модель комбинирует некогерентное рассеяние на поверхности коротковолновых фононов и почти баллистическое распространение длинноволновых фононов.

Теплопроводность сверхрешеток значительно уступает теплопроводности составляющих их материалов.

При дальнейшем уменьшении размеров нанопроволока переходит в молекулярную цепь, а тонкие пленки - в молекулярные полосы. Знаменитый численный эксперимент Ферми, Пасты и Улама показал, что теплопроводность длинной цепи взаимодействующих частиц может расходиться с длиной цепи как положительная степень длины цепи в одномерном случае и демонстрировать логарифмическую расходимость в двумерных задачах [100] для так называемых интегрируемых систем (цепь FPU, неупорядоченная гармоническая цепь, одномерный двухатомный газ, двухатомная решетка Тоды). Эта проблема и ее связь с экстремально высокой теплопроводностью нанотрубок из углерода и нитрида бора [101,102] и полос графена не рассматриваются в настоящем обзоре. Эти вопросы детально обсуждены в обзорах S. Lepri, R. Livi, A. Politi, Thermal conduction in classical lowdimensional lattices, Phys. Rep., 377 (1), 1 (2003), S.R. Xie, G. Zhang, B. Li Liu, X. Xu, Anomalous heat conduction and anomalous di usion in low dimensional nanoscale systems,
Eur. Phys. J. B, 85, 337 (2012) и монографии S. Lepri, Thermal Transport in Low Dimensions: from Statistical Physics to Nanoscale Heat Transfer, Lecture Notes in Physics Book 921. (Springer, 2016).

Следует помнить, что температура в точке строго определяется при наличии локального равновесия, поэтому можно достоверно рассматривать разность температур между точками, разделенными расстоянием не меньше длины свободного пробега носителей энергии [103,104].

Разработан ряд обобщающих закон Фурье моделей путем модификации определяющего соотношения между градиентом температуры и тепловым потоком. Большинство этих моделей учитывают временную нелокальность („память“материала), некоторые включают эффекты пространственной нелокальности для материалов с внутренней структурой. Такахаши [105] отметил, что пространственная нелокальность связана с появление масштаба, промежуточного между микро- и макро- масштабами мезомасштаба.

\section{1. Модели с задержкой}

Общее соотношение для теплового потока можно записать как [106]:

$$
\mathbf{q}=\int_{-\infty}^{t} Q\left(t-t^{\prime}\right) \nabla T d t^{\prime},
$$

где $Q(s)$ - положительная убывающая функция, называемая релаксационным ядром типа Джеффри [107-109]), стремящаяся к нулю при $s \rightarrow \infty$. Для $Q(s)=\lambda \delta(s)$, где $\delta(s)$ - функция Дирака, мы получаем закон Фурье (1).

Различный выбор определяющего соотношения приводит к различным моделям „с задержкой (см. $[59,64,110,111]$ и цитированную там литературу).

\section{1. Уравнение Максвелла-Каттанео-Вернотта}

Определяющее соотношение для теплового потока типа Джеффри имеет вид $[107,112]$ :

$$
\tau \frac{\partial \mathbf{q}}{\partial t}+\mathbf{q}=-\lambda \nabla T-\tau \lambda_{1} \frac{\partial}{d t} \nabla T
$$

Для случая $\lambda_{1}=0$ уравнение (4) сводится к хорошо известному уравнению Каттанео (также называемому уравнением Максвелла-Каттанео-Вернотта) определяющему соотношению, полученному независимо Морсом и Фешбахом (1953), Грэдом (1958) и Верноттом (1958)

$$
\tau \frac{\partial \mathbf{q}}{\partial t}+\mathbf{q}=-\lambda \nabla T
$$

и, если дополнительно $\tau=0-$ к закону Фурье. 
Уравнение Каттанео может быть получено в рамках расширенной неравновесной термодинамики [1,113], которая рассматривает диссипативные потоки, такие, как поток тепла, основные независимые переменные. Таким образом, энтропия зависит от внутренней энергии и теплового потока $s=s(u, q)$ и следует эволюционному уравнению

$$
\frac{\partial s}{\partial t}+\nabla \mathbf{J}^{s}=\sigma^{s} \geq 0
$$

где $\mathbf{J}^{s}-$ поток энтропии, $\sigma^{s}-$ скорость производства энтропии.

Определение неравновесной температуры как $T^{-1}=\partial s / \partial u$ и предположение, что $\partial s / \partial q=-\alpha T$, где $\alpha$ - материальный коэффициент, приводит (с учетом баланса энергии для находящегося в покое твердого тела) к уравнению

$$
\frac{\partial s}{\partial t}=-\nabla \cdot \frac{\mathbf{q}}{T}+\mathbf{q} \cdot\left(\nabla T^{-1}-\alpha \frac{\partial}{\partial t}\right),
$$

и, таким образом:

$$
\sigma^{s}=\mathbf{q} \cdot\left(\nabla T^{-1}-\alpha \frac{\partial \mathbf{q}}{\partial t}\right)
$$

Простейший способ обеспечить положительность производства энтропии $\sigma^{s}-$ предположить линейную связь между тепловым потоком и термодинамической силой (в скобках)

$$
\nabla T^{-1}-\alpha \frac{\partial \mathbf{q}}{\partial t}=\mu \mathbf{q},
$$

где $\mu-$ положительный коэффициент. Вводя обозначения $\alpha / \mu=\tau, \mu^{-1} \cdot T^{-2}=\lambda$, получим уравнение Каттанео.

Жоу и Касас-Вазуэз [114] показали, что подобным способом можно включить в уравнение Каттанео нелокальные члены, предполагая, что обобщенная энтропия, поток энтропии и производство энтропии явно зависят от теплового потока тензора $\hat{Q}$ :

$$
\tau \frac{\partial \mathbf{q}}{\partial t}+\mathbf{q}=-\lambda \nabla T+l^{2} \nabla^{2} \mathbf{q}
$$

Это уравнение отличается от уравнения Гюера-Крумхансла (см. ниже) отсутствием членов вида $\nabla \nabla \cdot \mathbf{q}$.

Время релаксации $\tau$ - время задержки, необходимое для установления стационарного потока тепла в объемном элементе, к которому приложен градиент температуры; временная задержка - результат „тепловой инерции“"

Тепловые возмущения в этой модели распространяются с конечной скоростью

$$
s=\sqrt{\frac{\lambda}{\rho C \tau}} .
$$

Оценки времени релаксации для твердых тел и разреженных газов могут быть записаны соответственно как [74]:

$$
\tau_{s}=\frac{3 \lambda}{\bar{c}^{2}}
$$

и

$$
\tau_{g}=\frac{3 v}{\bar{c}^{2}},
$$

где $\bar{c}-$ скорость фононов в твердом теле или средняя скорость молекул в газе

$$
\bar{c}=\sqrt{\frac{8}{\pi} \frac{k_{B} T}{m}},
$$

где $v-$ кинематическая вязкость газа, $m-$ масса молекулы.

Иногда используется число Каттанео, определяемое как

$$
C a=\frac{\kappa \tau}{L^{2}},
$$

где $\kappa=\lambda(\rho C)$ - температуропроводность.

Определяющее соотношение Каттанео (5) можно рассматривать как результат разложения в ряд Тэйлора:

$$
\mathbf{q}(\mathbf{r}, t+\tau)=-\lambda \nabla T(\mathbf{r}, t),
$$

называемое иногда „улучшенным“соотношением Каттанео или моделью с одиночной задержкой (single-phase lag - SPL-модель) [18]. Чен и др. [115] вывели уравнения SPL модели из уравнения Больцмана, используя для производной по времени аппроксимацию

$$
\frac{\partial f}{\partial t} \approx \frac{f(t+\Delta t)}{\Delta t}=\frac{f(t+\tau)}{\tau} .
$$

Недавно Ли и Цао [116] отметили, что модель Каттанео не следует рассматривать как частный случай SPLмодели, поскольку величина отброшенных членов разложения Тэйлора неизвестна и может быть значительной.

Определяющее соотношение Каттанео (5) можно представить как интеграл градиента температуры

$$
\mathbf{q}=-\frac{\lambda}{\tau} \int_{-\infty}^{t} \exp \left(-\frac{t-t^{\prime}}{\tau}\right) \nabla T d t^{\prime}
$$

Таким образом, модифицированное уравнение Фурье (уравнение Каттанео) можно записать (для случая постоянных свойств) как $[117,118]$

$$
\frac{\partial T}{\partial t}+\tau \frac{\partial^{2} T}{\partial t^{2}}=\frac{\lambda}{\rho c} \nabla^{2} T .
$$

Это уравнение можно рассматривать как частный случай телеграфного уравнения.

Франкел и др. [119] заметили, что альтернативная формулировка — в терминах теплового потока (скалярные уравнения для трех компонент в общем случае) может быть полезна для задач с граничными условиями, включающими тепловой поток. Распределение температуры в этом случае находится интегрированием уравнения сохранения энергии

$$
T(t)=T(0)+\int_{0}^{t} \frac{1}{\rho c}\left[-\nabla \cdot q\left(t^{\prime}\right)+Q\right] d t^{\prime} .
$$


Нир и Цао [120] сравнили три группы численных методов, использующих различные представления через температуру, тепловой поток и гибридное представление, и нашли последний способ предпочтительным.

Иногда уравнение Каттанео называют демпфированной версией уравнения Фурье [121].

Модель Каттанео устраняет парадокс бесконечной скорости распространения возмущений, но вводит новый: уравнение Каттанео не является инвариантным относительно преобразований Галилея: скорость распространения возмущений в системе, движущейся со скоростью $U$, являются нелинейной функцией $U$ [122]:

$$
c_{1,2}=\frac{1}{2}\left[U \pm \sqrt{U^{2}+4}\right]
$$

Этот парадокс устраняется, когда вместо частной производной по времени используется материальная производная [122]. Позднее Христов [123] предложил использовать независящую от системы координат конвективную производную Олдройда [124] и записывать уравнение Каттанео в виде

$$
\tau\left[\frac{\partial \mathbf{q}}{\partial t}+\mathbf{v} \cdot \nabla \mathbf{q}+\mathbf{q} \cdot \nabla \mathbf{v}+(\nabla \cdot \mathbf{v}) \mathbf{q}\right]+\mathbf{q}=-\lambda \nabla T .
$$

Джозеф и Презиози [107] предложили записывать релаксационное ядро в виде $R_{J P}=\lambda_{1} \delta(s)+$ $+\left(\lambda_{2} / \tau\right) \exp (-s / \tau)$, где $\lambda_{1}-$ эффективная теплопроводность и $\lambda_{2}-$ упругая теплопроводность. В этом случае тепловой поток (в одномерном приближении)

$$
q=-\lambda_{1} \frac{\partial T}{\partial x}-\frac{\lambda_{2}}{\tau} \int_{-\infty}^{t} \exp \left(\frac{t-s}{\tau}\right) \frac{\partial T}{\partial x} d s .
$$

Барлетта и Занчини [125-127] проанализировали совместимость уравнения Каттанео со вторым законом термодинамики и рассмотрели парадокс Тайфеля (превышение температурой граничных значений для слоя, поверхности которого имеют различную температуру) и обнаружили, что производство энтропии может быть отрицательным в областях, где тепловой поток уменьшается быстрее, чем $\partial \mathbf{q} / \partial t|>| \mathbf{q} \mid / \tau$. Однако этот результат нельзя рассматривать как нарушение второго закона классической термодинамики, основанной на гипотезе локального равновесия, которая не выполняется [128]. Закон Каттанео совместим со вторым законом в расширенной неравновесной термодинамике $[129,130]$.

Ли и Цао [131] изучали термодинамические проблемы SPL-модели. Используя выражение для скорости производства энтропии

$$
S=-\frac{\mathbf{q} \cdot \nabla T}{T^{2}}
$$

классической неравновесной термодинамики, авторы получили для уравнения Фурье

$$
S_{F}=-\frac{q^{2}}{\lambda T^{2}} \geq 0
$$

и для SPL-модели

$$
S_{S P L}^{C I T}=-\frac{\mathbf{q}(t) \cdot \mathbf{q}(t+\tau)}{\lambda T^{2}} .
$$

Очевидно, что скорость производства энтропии для SPL-модели не обязательно положительна или равна нулю. Второй закон удовлетворяется в расширенной неравновесной термодинамике [129], в которой скорость производства энтропии равна

$$
S_{S P L}^{E I T}=-\frac{\mathbf{q}(t+\tau) \cdot \mathbf{q}(t+\tau)}{\lambda T^{2}} .
$$

В биологических задачах уравнение Каттанео (с добавлением источниковых членов из уравнения Пеннеca (2),(3)) часто называется моделью тепловой волны [132], иногда используется термин „температурная волна“ $[133]$.

\section{2. Модели с двойной задержкой}

Для учета как эффектов релаксации, так и микроструктуры вводятся модели с двойной задержкой (DPLмодели) [134-136]

$$
\mathbf{q}\left(\mathbf{r}, t+\tau_{q}\right)=-\lambda \nabla T\left(\mathbf{r}, t+\tau_{T}\right),
$$

где $\tau_{q}$ и $\tau_{T}$ - времена задержки для теплового потока и градиента температуры, возникающие вследствие „тепловой инерции“ и „"микроструктурных взаимодействийб $[137]$.

DPL-модель сводится к SPL-модели (модели с одиночной задержкой) при $\tau_{T}=0$ и к закону Фурье при $\tau_{T}=\tau_{q}=0$.

Оба релаксационных времени чрезвычайно малы для обычных материалов. Например, $\tau_{q}$ и $\tau_{T}$ для золота составляют 8.5 и 90 ps соответственно [138].

В опубликованных данных по времени релаксации в биологических тканях много противоречий. Эти времена составляют от 14-16 до 0.043-0.056 s для обработанного мяса [78], эксперименты с мышцами коровы дают значения $7.36-8.43$ и $14.54-21.03$ s. [139]. Жанг [140] изучал релаксационное время в зависимости от свойств тканей и крови и ввел межфазный конвективный коэффициент.

Уравнение (6) может быть переписано через разность релаксационных времен

$$
\mathbf{q}(\mathbf{r}, t)=-\lambda \nabla T\left(\mathbf{r}, t+\left(\tau_{T}-\tau_{q}\right)\right) .
$$

Таким образом, DPL-модель не зависит от релаксационных времен $\tau_{T}$ и $\tau_{q}$ по отдельности, а только от их разности [141], и SPL- и DPL-модели математически эквивалентны [142].

DPL-модель близка [143] к гиперболическим моделям, описывающим обмен энергией между электронами и фононами [144-146], которые в одномерном случае имеют 
вид

$$
\begin{gathered}
c_{e} \frac{\partial T_{e}}{\partial t}=-\frac{\partial q}{\partial x}-G\left(T_{e}-T_{l}\right)+Q, \\
c_{l} \frac{\partial T_{l}}{\partial t}=G\left(T_{e}-T_{l}\right), \\
\tau \frac{\partial q}{\partial t}+\lambda \frac{\partial T_{e}}{\partial x}+q=0,
\end{gathered}
$$

где $T_{e}$ - температура электронного газа, $T_{l}$ - температура решетки, $c_{e}$ и $c_{l}$ - теплоемкости электронного газа и решетки соответственно, $G$ - коэффициент связи электрон-фонон.

Тзоу [147] оценил релаксационные времена DPLмодели через параметры $G, c_{e}, c_{l}$ и получил для меди, серебра, золота и свинца значения $\tau_{T}$ и $\tau_{q}$ около $10^{-11}$ и $10^{-13}$ соответственно.

Жанг [148] предложил явные выражения для теплообмена в тканях

$$
\begin{aligned}
\tau_{q} & =\frac{\varepsilon(1-\varepsilon)}{\frac{\varepsilon}{C_{t b}}+(1-\varepsilon)} \frac{\rho_{b} C_{b}}{G}, \\
\tau_{T} & =\frac{\varepsilon(1-\varepsilon)}{\frac{\varepsilon}{K_{t b}}+(1-\varepsilon)} \frac{\rho_{b} C_{b}}{G},
\end{aligned}
$$

где $C_{t b}=\left(\rho_{t} C_{t}\right) /\left(\rho_{b} C_{b}\right)$ - отношение теплоемкостей тканей и крови, $K_{t b}=\lambda_{t} / \lambda_{b}-$ отношение теплопроводностей, $\varepsilon-$ пористость тканей, $G-$ конвективноперфузионный параметр.

Тзоу и Дай [149] рассмотрели задержки в системе со многими носителями. Уравнения для системы с $\mathrm{N}$ носителями записываются как

$$
\begin{gathered}
C_{1} \frac{\partial T_{1}}{\partial t}=\lambda_{1} \nabla^{2} T_{1}-\sum_{j=2}^{N} G_{1 i}\left(T_{1}-T_{i}\right) \\
C_{m} \frac{\partial T_{m}}{\partial t}=\lambda_{m} \nabla^{2} T_{m}+\sum_{j=1}^{m-1} G_{j m}\left(T_{j}-T_{m}\right)-\sum_{i=m+1}^{N} G_{m i}\left(T_{m}-T_{i}\right), \\
m=2,3, \ldots(N-1), \\
C_{N} \frac{\partial T_{N}}{\partial t}=\lambda_{N} \nabla^{2} T_{N}+\sum_{i=1}^{N-1} G_{i N}\left(T_{i}-T_{N}\right) .
\end{gathered}
$$

При выводе уравнения для единой температуры в системе трех носителей (например, композитный материал с тремя компонентами или полярные полупроводники, в которых энергия может переноситься электронами, дырками и фононами) Тзоу и Дай обнаружили нелинейные эффекты, связанные с $\tau_{q}^{2}$ и $\tau_{t}^{2}$.

Использование первых членов разложения по $\tau_{q}$ и $\tau_{T}$ дает

$$
q+\tau_{q}+\frac{\partial q}{\partial t}=-\lambda\left[\nabla T+\tau_{T} \frac{\partial T}{\partial t}\right] .
$$

Использование этого соотношения в законе сохранения энергии дает DPL-модель типа I [79,150] (также линейная DPL-модель [151]).
Уравнения модели можно переписать в терминах теплового потока вместо температуры и даже в терминах потенциала теплового потока, определяемого как $\mathbf{q}=\nabla \phi[152]$.

Ванг и др. [152] доказали корректность DPL-модели на одномерном интервале с однородными граничными условиями Дирихле, Неймана или Робина. Позднее Ванг и Ху [153] обобщили этот результат на $n$-мерный случай.

DPL-модель типа II получается, если использованы разложения Тейлора первого и второго порядка для $q$ и $T$ соответственно

$$
q+\tau_{q} \frac{\partial q}{\partial t}=-\lambda\left[\nabla T+\tau_{T} \frac{\partial \nabla T}{\partial t}+\frac{\tau_{T}}{2} \frac{\partial^{2} \nabla T}{\partial t^{2}}\right],
$$

и типа III (DPL-модель второго порядка [154]) — когда для $q$ и $T$ использованы разложения второго порядка

$$
\begin{aligned}
& q+\tau_{q} \frac{\partial q}{\partial t}+\frac{\tau_{q}}{2} \frac{\partial^{2} q}{\partial t^{2}} \\
& =-\lambda\left[\nabla T+\tau_{T} \frac{\partial \nabla T}{\partial t}+\frac{\tau_{T}}{2} \frac{\partial^{2} \nabla T}{\partial t^{2}}\right] .
\end{aligned}
$$

Иногда используется иная нотация для различения DPL-моделей с указанием порядков разложения, напримep DPL (2,1) [155]. Руколайне [156] установил, что решение DPL-модели неустойчиво. Позднее он подтвердил это заключение для DPL-модели типа III [157].

Квинтанилла и Раке [158] (см. также [159]) проанализировали устойчивость решений различных версий DPL-модели. Авторы ввели параметр, контролирующий устойчивость решения, как отношение времен релаксации DPL-модели

$$
\xi=\frac{\tau_{T}}{\tau_{q}} .
$$

Авторы рассмотрели характеристический полином, связанный с преобразованием Лапласа в ограниченной области для случая условий Дирихле. Поведение решения определяется действительной частью собственного значения.

Результаты исследования можно суммировать как

- если использовано приближение первого порядка по $\tau_{q}$ и первого или второго по $\tau_{T}$, система всегда устойчива;

- если использовано приближение второго порядка по $\tau_{q}$ и первого по $\tau_{T}$, система устойчива, если $\xi>1 / 2$, и неустойчива, если $\xi<1 / 2$;

- если использовано приближение второго порядка по $\tau_{q}$ и $\tau_{T}$, система устойчива, когда $\xi>2-\sqrt{3}$, и неустойчива, если $\xi>2-\sqrt{3}$;

- если $\xi>1 / 2$, то все типы DPL-моделей ведут себя одинаково.

Условия на отношение $\xi=\frac{\tau_{T}}{\tau_{q}}$ были также выведены Фабрицио и Лаззари из второго закона термодинамики [160].

Совместимость DPL-модели со вторым законом термодинамики в расширенной необратимой термодинамике доказал Ху [161]. 
Как модель тепловой волны, так и DPL-модель активно используются для расчета теплопереноса в биологических задачах. Например, воздействие лазерного излучения на биологические ткани исследовали Жоу и др. [162], Яних и др. [163], Африн и др. [164], Ахмадикиа и др. [165], Саху и др. [166], Лиу и Ванг [167], Пур и др. [168], Хухманд и др. [169], Кумар и Сривастава [170], Ясински и др. [171]. Жоу и др. [172] решили двумерную (осесимметричную) задачу для двух случаев - поверхностный нагрев и объемный нагрев; авторы нашли многомерные эффекты существенными.

Норузи и др. использовали модель тепловой волны для изучения теплопереноса в полосе при нагреве лазерным излучением и DPL-модель для объемного нагрева полосы [173].

Лиу и др. [132] вычислили изменение температуры при ультразвуковом воздействии в рамках модели тепловой волны. Ли и др. [174] использовали DPL-модель для расчета температурного отклика ex vivo при воздействии сфокусированного ультразвукового нагрева на гомогенный фантом биологической ткани и на гетерогенную ткань печени.

Кумар и др. применили конечноэлементный вейвлетметод Галеркина для исследования гипертермии в предположении гауссова характера внешнего источника тепла [175].

Хо и др. [176] применили метод решетки Больцмана для решения DPL-модели теплопереноса в двуслойной системе.

Моради и Ахмадикиа [177] использовали DPL-модель для расчета теплопереноса при сверхбыстром замораживании биологических тканей (скорость охлаждения около $1000^{\circ} \mathrm{s}$ [178]) когда в замороженной области формируется аморфный лед [44].

Лиу и Чен [179] исследовали гипертермию с применением магнитной жидкости в рамках DPL-модели.

Борялило и др. применили DPL-модель для решения задачи термоупругости - демпфированные колебания микробалки [180].

Чоу и Янг [181] исследовали в двумерной постановке теплоперенос в многослойной структуре с использованием метода пространственно-временных консервативных элементов [182]. Этот метод был разработан для решения уравнений Эйлера и Навье-Стокса [183] и обеспечивает локальное и глобальное сохранение потока. Авторы установили разные режимы теплообмена: гиперболический, волнообразный, диффузионный, сверхдиффузионный.

\section{3. Модель с тройной задержкой}

Модель с тройной задержкой получается из DPLмодели введением дополнительно к временам релаксации для теплового потока и градиента температуры релаксационного времени для градиента теплового сме- щения ${ }^{3}[110,187-189]$

$$
\mathbf{q}\left(\mathbf{r}, t+\tau_{q}\right)=-\left[\lambda \nabla T\left(\mathbf{r}, t+\tau_{T}\right)+C c \nabla v\left(r, t+\tau_{v}\right)\right]
$$

Модель с тройной задержкой используется также для анализа задач термоупругости (см., например, [190]).

\section{2. Фононные модели}

Фононы - квантованные колебания решетки (упругие волны могут существовать только для определенных значений энергии). Фононы являются носителями энергии в диэлектрических и полупроводниковых кристаллах. Следует рассматривать различные механизмы рассеяния фононов: нормальное ( $N$-процесс), Umklapp $(U$-процесс), рассеяние на дефектах решетки, рассеяние на границах.

Функция распределения фононов описывается уравнением Больцмана, имеющим вид

$$
\frac{\partial f}{\partial t}+\mathbf{v} \nabla f+\mathbf{F} \frac{\partial f}{\partial \mathbf{P}}=\left(\frac{\partial f}{d t}\right)_{s c a t t},
$$

где $\mathbf{P}$ - импульс, $\mathbf{F}-$ внешняя сила.

Часто для линеаризации уравнения Больцмана используется следующая аппроксимация столкновительного члена (приближение времени релаксации)

$$
\left(\frac{\partial f}{d t}\right)_{s c a t t}=-\frac{f-f_{0}}{\tau},
$$

где $f_{0}$ - равновесное распределение.

Широко распространено приближение Каллавея [6,96]

$$
\left(\frac{\partial f}{d t}\right)_{\text {scatt }}=-\frac{f-f_{\lambda}}{\tau_{N}}-\frac{f-f_{0}}{\tau_{R}},
$$

где $f_{\lambda}-$ функция распределения однородно дрейфующего фононного газа, $\tau_{N}$ - время релаксации $N$-процесса и $\tau_{R}$ - время релаксации $U$-процесса.

Наличие фононов с широким спектром означает отсутствие единого значения средней длины свободного пробега фононов, которое определяет режим теплопереноса.

Вычисления на основе первых принципов показывают различные распределения для разных материалов. Например, в кремнии 80\% тепла переносится фононами с длиной свободного пробега от 0.05 до $8 \mu \mathrm{m}$, а в алмазе $80 \%$ тепла переносится фононами с длиной свободного пробега от 0.3 до $2 \mu \mathrm{m}$ [50]; более 95\% тепла в сапфире переносится фононами с длиной свободного пробега короче $1 \mu \mathrm{m}[39]$.

\footnotetext{
${ }^{3}$ Тепловое смещение было введено Р. фон Гельмгольцем[184]. Оно удовлетворяет условию $\dot{v}=T$. Эта величина была использована Грином и Нагхди[185,186] как „скалярная историческая переменная“ $v=\int_{0}^{t} T(\tau) d \tau+v_{0}$
} 


\section{1. Уравнение Гюйера-Крумхансла}

Гюйер и Крумхансл $[191,192]$ решили линеаризованное уравнение Больцмана, предполагая, что скорость нормального рассеяния много больше скорости $U$-процессов при низких температурах. Они предложили феноменологическую связь между фононами и колебаниями решетки, связанными с ее ангармоничностью.

Когда средняя длина свободного пробега фононов превышает размер образца, поведение фононного газа становится подобным кнудсеновскому течению или баллистическому переносу. Авторы определили условия, при которых течение Пуазейля вносит существенный вклад в теплопроводность.

Течение Пуазейля в цилиндре описывается уравнением [114]

$$
\Lambda^{2} \nabla^{2} \mathbf{q}=\lambda T
$$

решение есть $q(r)=A\left(R^{2}-r^{2}\right), A=-\left(\lambda \nabla T /\left(\Lambda^{2}\right)\right)$.

Обычно уравнение Гюйера-Крумхансла записывается в виде

$$
\tau \frac{\partial \mathbf{q}}{\partial t}+\mathbf{q}=-\lambda \nabla T+\beta^{\prime} \Delta \mathbf{q}+\beta^{\prime \prime} \nabla \operatorname{cdot} \nabla \mathbf{q},
$$

где $\beta^{\prime}$ и $\beta^{\prime \prime}-$ коэффициенты Гюйера-Крумхансла (в случае разреженного газа эти коэффициенты связаны с интегралом Каллавея [22]) или

$$
\tau \frac{\partial \mathbf{q}}{\partial t}+\mathbf{q}=-\lambda \nabla T+\Lambda^{2}\left(\nabla^{2} \mathbf{q}+2 \nabla \cdot \nabla \mathbf{q}\right),
$$

где $\Lambda$ - средняя длина свободного пробега фононов.

Уравнение Гюйера-Крумхансла может быть получено в расширенной неравновесной термодинамике в предположении, что нелокальные члены могут быть включены в выражение для потока энтропии как [1]

$$
\mathbf{J}^{s}=\frac{\mathbf{q}}{T}+\gamma(\mathbf{q} \cdot \nabla \mathbf{q}+2 \mathbf{q} \nabla \cdot \mathbf{q}),
$$

где $\gamma$ - положительный коэффициент.

Недавно Кальво-Шварцвальдер и др. [193] использовали уравнение Гюйера-Крумхансла для решения одномерной задачи Стефана.

\section{2. Баллистико-диффузионная модель}

Введенная Ченом [194,195] баллистико-диффузионная модель основана на расщеплении функции распределения (а также внутренней энергии и теплового потока) на две компоненты

$$
f=f_{b}+f_{d}, \quad e=e_{b}+e_{d}, \quad \mathbf{q}=\mathbf{q}_{b}+\mathbf{q}_{d},
$$

отражающем сосуществование двух типов носителей тепла:

- баллистические фононы, которые рассеиваются главным образом на границах,

- диффузионные фононы, испытывающие многочисленные акты рассеяния в объеме системы.
Относительная роль этих компонент определяется значением числа Кнудсена и геометрией системы [196].

Янг и др. [197] использовали уравнение Больцмана относительной интенсивности фононов $I_{\omega}=$ $=\mathbf{v}_{\omega} \hbar \omega f D(\omega) /(4 \pi)\left(\mathbf{v}_{\omega}-\right.$ групповая скорость носителей, $\omega$ - частота фононов, $D(\omega)$ - плотность состояний фононов в единице объема, $S_{\omega}-$ источниковый член, который может определяться, например, электронфононным рассеянием)

$$
\frac{\partial I_{\omega}}{\partial t}+\mathbf{v}_{\omega} \nabla I_{b \omega}=-\frac{I_{\omega}-I_{0 \omega}}{\tau_{\omega}}+S_{\omega}
$$

Уравнения для баллистической и диффузионной частей записываются соответственно как

$$
\frac{\partial I_{b \omega}}{\partial t}+\mathbf{v}_{\omega} \nabla I_{b \omega}=-\frac{I_{b \omega}}{\tau_{\omega}}+S_{\omega}
$$

и

$$
\frac{\partial I_{d \omega}}{\partial t}+\mathbf{v}_{\omega} \nabla I_{d \omega}=-\frac{I_{d \omega}-I_{0 \omega}}{\tau_{\omega}}+S_{\omega} .
$$

Аллен [198] провел анализ перехода от баллистического режима к диффузионному, используя компьютерное моделирование и версию уравнения Больцмана, подвергнутого преобразованию Фурье. Эволюционные уравнения для средней заселенности в обратном пространстве фононной моды $Q$ включают ряд членов (дрейф, рассеяние, внешний)

$$
\begin{gathered}
\frac{\partial N_{Q}}{\partial t}=\left(\frac{d N_{Q}}{d t}\right)_{d r i f t}+\left(\frac{d N_{Q}}{d t}\right)_{s c a t t}+\left(\frac{d N_{Q}}{d t}\right)_{\text {ext }}, \\
\left(\frac{d N_{Q}}{d t}\right)_{d r i f t}=-\mathbf{v}_{Q} \cdot \nabla N_{Q}=-\mathbf{v}_{Q}\left[\frac{d n_{Q}}{d T} \nabla T+\nabla \Phi_{Q}\right], \\
\left(\frac{d N_{Q}}{d t}\right)_{s c a t t}=-\sum_{Q^{\prime}} S_{Q, Q^{\prime}} \Phi_{Q^{\prime}},
\end{gathered}
$$

где $n_{Q}$ - локальное равновесное распределение Бозе-Эйнштейна, $\Phi_{Q}=N_{Q}-n_{Q}, S_{Q, Q^{\prime}}$ - линеаризованный оператор рассеяния.

Васкуез и др. [36] и Лебон и др. [1] рассмотрели двухтемпературный вариант баллистикодиффузионной модели. Васкуез и др. использовали уравнение Гюйера-Крумхансла для описания баллистического и диффузионного тепловых потоков. Лебон и др. использовали уравнение Гюйера-Крумхансла только для баллистического переноса; для описания диффузионной части применялось уравнение Каттанео.

О непосредственном наблюдении баллистического и диффузионного переноса в графене сообщили Пумрол др. [95].

\section{3. Обобщенный закон Фурье Хуа и др.}

Хуа и др. [199] разработали обобщенный закон Фурье, справедливый от баллистического до диффузионного режима на основе помодового уравнения 
Больцмана в приближении времени релаксации (модель Бхатнагара-Гросса-Кука)

$$
\frac{\partial g_{\mu}(x, t)}{\partial t}+\mathbf{v}_{\mu} \cdot g_{\mu}(\mathbf{x}, t)=\frac{g_{\mu}-g_{0}(T, \mathbf{x}, t)}{\tau_{\mu}}+\dot{Q}_{\mu},
$$

где $g_{\mu}(\mathbf{x}, t)=\hbar \omega_{\mu}\left(f_{\mu}(\mathbf{x}, t)-f_{0}(T)\right)-$ функция распределения отклонения энергии для фонона в состоянии $\mu=(\mathbf{q}, s), \mathbf{q}-$ волновой вектор фонона, $s-$ индекс ветви фононов, $f_{0}-$ распределение Бозе-Эйнштейна, $\left.g_{0}(T)=\hbar \omega_{\mu} f_{0}(T)-f_{0}\left(T_{0}\right)\right) \approx C_{\mu} \Delta T, C_{\mu}-$ удельная теплоемкость, зависящая от моды, $\dot{Q}-$ скорость поступления энергии в расчете на моду.

Для решения этого уравнения авторы использовали преобразование Фурье по времени и связали температурный градиент с зависящей от моды теплоемкостью $\mathbf{x}$.

\section{4. Фононная гидродинамика}

Гуо и Ванг [200] получили макроскопические уравнения движения фононного газа на основе уравнения Больцмана для фононов

$$
\frac{\partial f}{\partial t}+\mathbf{v}_{g}=C(f)
$$

где $f=f(\mathbf{x}, t, \mathbf{k})-$ функция распределения фононов, $\mathbf{v}_{g}=\nabla_{\mathbf{k}} \omega-$ групповая скорость фононов.

Столкновительный член $C(f)$ включает вклад двух основных процессов рассеяния: нормальное рассеяние $(N$ процесс) и рассеяние с потерей импульса ( $R$-процесс).

Энергия сохраняется в процессах рассеяния любого типа, квазиимпульс - только при нормальном рассеянии.

Упрощение уравнения Больцмана основано на использовании модели релаксационного времени Каллавея, которая предполагает, что $N$-процесс и $R$-процесс протекают независимо. При низких температурах доминирующим является $N$-процесс, однако при обычных температурах $N$-процессом можно пренебречь, и уравнение Больцмана принимает вид

$$
\frac{\partial f}{\partial t}+\mathbf{v}_{g}=-\frac{f-f_{R}^{e q}}{\tau_{R}},
$$

где $f_{R}^{e q}-$ равновесная функция распределения для $R$-процесса.

Модель фононной гидродинамики использует полевые переменные:

плотность энергии фононов

$$
e=\int \hbar \omega f d \mathbf{k}
$$

тепловой поток

$$
\mathbf{q}=\int \hbar \omega \mathbf{v}_{g} f d \mathbf{k}
$$

поток теплового потока

$$
\hat{Q}=\int \hbar \omega \mathbf{v}_{g} \mathbf{v}_{g} f d \mathbf{k}
$$

Интегрирование уравнения Больцмана в пространстве волновых векторов дает уравнения баланса для плотности энергии

$$
\frac{\partial e}{\partial t}+\nabla \cdot \mathbf{q}=0
$$

и теплового потока

$$
\frac{\partial \mathbf{q}}{\partial t}+\nabla \cdot \hat{Q}=-\frac{\mathbf{q}}{\tau_{R}} .
$$

Эти уравнения баланса - четырехмоментные полевые уравнения для фононного уравнения Больцмана. ${ }^{4}$ Для замыкания системы уравнений переноса для фононов поток теплового потока $\hat{Q}$ должен быть определен в терминах четырех основных полевых переменных (плотность энергии и три компоненты теплового потока).

Известен ряд подходов к проблеме замыкания в кинетической теории:

1) метод Гилбета,

2) разложение Чепмана-Энскога,

3) метод моментов Грэда,

4) „регуляризованный метод моментов“ (R13-метод),

5) метод инвариантного многообразия.

Авторы использовали метод возмущений относительно равновесной четырехмоментной функции распределения фононов, полученной с помощью принципа максимума энтропии.

Проблема свелась, таким образом, к максимизации следующего функционала:

$$
\begin{aligned}
& \Phi=-k_{B} \int[f \ln (f)-(1+f) \ln (1+f)] d \mathbf{k} \\
& +\beta\left(e-\int \hbar \omega f d \mathbf{k}\right)+\gamma_{i}\left(q_{i}-\int v_{g i} \hbar \omega f d \mathbf{k}\right),
\end{aligned}
$$

где $\beta$ и $\gamma_{i}-$ множители Лагранжа.

В итоге четырехмоментная функция распределения фононов имеет вид

$$
f_{4}=\frac{1}{\exp \left(\beta \frac{\hbar \omega}{k_{B}}+\gamma_{i} \frac{\hbar \omega}{k_{B}}\right)-1} .
$$

Приближения более высокого порядка для потока теплового потока $\hat{Q}$ находятся из уравнения баланса

$$
\frac{\partial Q_{i j}}{\partial t}+\frac{\partial M_{i j k}}{\partial x_{k}}=\frac{1}{\partial \tau_{R}}\left(\frac{1}{3} v_{g}^{2} e f_{i j}-Q_{i j}\right)
$$

тензор третьего порядка $\hat{M}$ определяется как

$$
M_{i j k}=\int v_{g i} v_{g j} v_{g k} \hbar \omega f d \mathbf{k} .
$$

Используется разложение по малому параметру числу Кнудсена $\varepsilon=\mathrm{Kn}$

$$
Q_{i j}=Q_{i j}^{(0)}+\varepsilon Q_{i j}^{(1)}+\ldots
$$

\footnotetext{
${ }^{4}$ Имеется опечатка в работе $[200]-\hat{Q}$ представлен как вектор.
} 
и сохраняются члены нулевого и первого порядков

$$
\begin{aligned}
Q_{i j} & =\frac{1}{3} v_{g}^{2} \varepsilon \delta_{i j}+\frac{2}{15} \tau_{R} v_{g}^{2} \frac{\partial q_{k}}{\partial x_{k}} \delta_{i j} \\
& -\frac{1}{5} \tau_{R} v_{g}^{2}\left(\frac{\partial q_{i}}{\partial x_{j}}+\frac{q_{j}}{\partial x_{i}}\right) .
\end{aligned}
$$

Окончательно уравнение баланса имеет вид

$$
\tau_{R} \frac{\partial \mathbf{q}}{\partial t}+\mathbf{q}=-\lambda \nabla T+\frac{1}{5} \Lambda^{2}\left[\nabla^{2} \mathbf{q}+\frac{1}{3} \nabla(\nabla \cdot \mathbf{q})\right],
$$

где $\Lambda=v_{g} \tau R-$ средняя длина свободного пробега.

Это уравнение отличается только численным коэффициентом при нелокальном члене от уравнения Гюйера-Крумхансла. Авторы подчеркивают, что математическая структура этих уравнений совпадает, однако лежащие в основе физические механизмы переноса различны. Нелокальный член в уравнении Гюйера-Крумхансла отражает нормальное рассеяние фононов - это уравнение предназначено для описания теплопереноса при низких температурах. Нелокальный член в уравнении фононной гидродинамики есть следствие пространственных неравновесных эффектов, возникающих из-за рассеяния фононов на границах или значительных градиентов температуры.

Гуо и Ванг использовали выведенные уравнения фононной гидродинамики для решения ряда задач:

- транспорт фононов в плоскости тонкой пленки;

- транспорт фононов в нанопроволоке;

- нестационарный одномерный транспорт фононов поперек тонкой пленки;

- высокочастотный периодический нагрев полубесконечной поверхности;

- нестационарный теплоперенос в тепловой решетке.

\section{5. Модель релаксонов}

Недавно (2020) Симончелли и др. [12] использовали эволюцию релаксонов для вывода пары уравнений, описывающих связанные коллективные колебания решетки в диэлектрических кристаллах.

Понятие релаксона как коллективного неравновесного возбуждения кристаллической решетки, представляющего собой линейную комбинацию фононов, было введено Чапеллотти и Марзари [201]. Теплопроводность можно рассматривать как движение газа релаксонов.

Авторы исходят из линеаризованного уравнения Больцмана для фононов, имеющего вид

$$
\frac{\partial n_{\mu}}{\partial t}+\mathbf{v}_{\mu} \nabla n_{\mu}=-\frac{1}{V} \sum_{\mu^{\prime}} \Omega_{\mu \mu^{\prime}} \Delta n_{\mu^{\prime}} .
$$

Суммирование ведется по всем возможным состояниям фонона $\mu(\mu=(\mathbf{q}, s)$, где $\mathbf{q}$ меняется по зоне Бриллюена, а $s$ - по ветвям фонона), $\mathbf{v}_{\mu}-$ групповая скорость фононов, $V$ - объем, $\Omega_{\mu \mu^{\prime}}$ - линейный оператор рассеяния фононов, $\Delta n_{\mu}=n_{\mu}-\bar{n}_{\mu}$ - отклонение функции распределения фононов от равновесной, т. е. от распределения Бозе-Эйнштейна

$$
\frac{1}{\exp \left(\frac{\hbar \omega_{\mu}}{k_{B} T}\right)-1}
$$

где $\omega_{\mu}$ - частота фонона.

Поскольку распределение Бозе-Эйнштейна зависит от пространственных координат и времени только через температуру, уравнение может быть переписано в виде

$$
\begin{aligned}
& \frac{\partial \bar{n} \mu}{\partial T}\left(\frac{\partial T}{\partial t}+\mathbf{v}_{\mu} \cdot \nabla T\right)+\frac{\partial \Delta n_{\mu}}{\partial t}+\mathbf{v}_{\mu} \cdot \nabla \Delta n_{\mu} \\
& \quad=-\frac{1}{V} \sum_{\mu^{\prime}} \Omega_{\mu \mu^{\prime}} \Delta n_{\mu^{\prime}} .
\end{aligned}
$$

Решение этого уравнения в замкнутом виде возможно в приближении единого времени релаксации, когда упрощается столкновительный оператор [201]

$$
\frac{1}{V} \sum_{\mu^{\prime}} \Omega_{\mu \mu^{\prime}} \Delta n_{\mu^{\prime}} \approx \frac{\Delta n_{\mu}}{\tau_{\mu}^{S T A}} .
$$

Теплопроводность при использовании гармонического приближения для теплового потока

$$
q=\sum_{\mu} \hbar \omega_{\mu} v_{\mu} \Delta n_{\mu}
$$

записывается как

$$
\left(\lambda_{\mu}^{i j}\right)^{S M A}=\frac{1}{V} \sum_{\mu} C_{\mu} v_{\mu}^{i}\left(\Lambda_{\mu}^{j}\right)^{S M A},
$$

где $\left(\Lambda_{\mu}^{j}\right)^{S M A}$ - компонент средней длины свободного пробега фонона в направлении $j$.

Таким образом, теплопроводность обеспечивается фононами, переносящими удельную теплоемкость

$$
\frac{C_{\mu}}{k_{B} T^{2}} \bar{n}_{\mu}\left(\bar{n}_{\mu}+1\right)\left(\hbar \omega_{\mu}\right)^{2},
$$

движущимися со скоростью $v_{\mu}$ со средней длиной свободного пробега $\left(\Lambda_{\mu}^{j}\right)^{S M A}$ перед термализацией при рассеянии.

Чапеллотти и Марзари подчеркивают, что определение времени жизни фонона или средней длины свободного пробега не может использоваться вне предположения о едином времени релаксации, поскольку внедиагональные члены оператора рассеяния вносят связь между фононами, и термализация фонона не может описываться экспоненциальной релаксацией.

Оператор $\Omega$ может быть приведен к симметричной форме использованием преобразования

$$
\begin{gathered}
\bar{\Omega}_{\mu \mu^{\prime}}=\Omega_{\mu \mu^{\prime}} \sqrt{\frac{\bar{n}_{\mu^{\prime}}\left(\bar{n}_{\mu^{\prime}}+1\right)}{\bar{n}_{\mu}\left(\bar{n}_{\mu}+1\right)},} \\
\Delta \bar{n}_{\mu}=\frac{\Delta n_{\mu}}{\bar{n}_{\mu}\left(\bar{n}_{\mu}+1\right)} .
\end{gathered}
$$


Поскольку $\bar{\Omega}$ - действительная положительная матрица, ее можно привести к диагональному виду и найти собственные вектора $\theta_{\mu}^{\alpha}$ и собственные значения $1 / \tau_{\alpha}$ ( $\alpha$ - индекс собственного значения)

$$
\frac{1}{V} \sum_{\mu^{\prime}} \Omega_{\mu \mu^{\prime}}=\frac{1}{\tau_{\alpha}} \theta_{\mu}^{\alpha}
$$

Произвольный $\Delta \bar{n}_{\mu}$ можно представить как линейную комбинацию собственных векторов $\theta_{\mu}^{\alpha}$ :

$$
\Delta \bar{n}_{\mu}=\sum_{\alpha} f_{\alpha} \theta_{\mu}^{\alpha}
$$

Уравнение Больцмана можно записать в базисе собственных векторов $\theta^{\alpha}$ :

$$
\begin{aligned}
& \sqrt{\frac{C}{k_{B} T^{2}}}\left(\frac{\partial T}{\partial t}\langle 0 \mid \alpha\rangle+\Delta T+\mathbf{V}_{\alpha}\right) \\
& +\frac{\partial f_{\alpha}}{\partial t}+\sum_{\alpha^{\prime}} \mathbf{V}_{\alpha \alpha^{\prime}} \cdot \nabla f_{\alpha}=-\frac{f_{\alpha}}{\tau_{\alpha}} .
\end{aligned}
$$

Чепеллотти и Марзари вывели следующее уравнение для теплопроводности:

$$
\lambda^{i j}=\frac{-1}{V \nabla_{i} T} \sum_{\mu} \hbar \omega_{\mu} v_{\mu}^{j} \Delta n_{\mu}=\sum_{\alpha} C V_{\alpha}^{i} \lambda_{\alpha}^{j}
$$

Авторы также показали, что широко используемое для оценки времени релаксации в системах с различными механизмами рассеяния правило Маттиессена переоценивает теплопроводность.

Релаксоны обладают четностью и только нечетные дают вклад в теплопроводность. Четные определяют термическую вязкость [12]. Симончелли и др. вывели два связанных уравнения для температуры и дрейфовой скорости.

\section{3. Термомассовая модель}

Термомассовая модель основана на старой идее Толмана [202]: носители тепла обладают дуальностью масса-энергия и проявляют энергетические свойства в процессах преобразования энергии, а массовые - в процессах переноса [203].

Масса тепла определяется, согласно эквивалентности массы и энергии Эйнштейна [204-206]:

$$
E=M c^{2}=\frac{M_{0} c^{2}}{\sqrt{1-\frac{v^{2}}{c^{2}}}},
$$

где $E-$ тепловая энергия, $M_{0}-$ масса покоя, $v-$ скорость носителя тепла, $c$ - скорость света в вакууме, $M$ - релятивистская масса.

Если $v \ll c$, это уравнение упрощается

$$
E \approx\left(M_{0}+M_{k}\right) c^{2} .
$$

Здесь $M_{k}$ - дополнительная масса, индуцированная кинетической энергией. Термомасса (ТМ) $M_{h}$ - релятивистская масса внутренней энергии $U$ :

$$
M_{h}=\frac{U}{c^{2}}
$$

Термомасса чрезвычайно мала $\left(10^{-16} \mathrm{~kg}\right.$ для $1 \mathrm{~J}$ тепла) [203].

Плотность термомассы, содержащейся в среде [207]:

$$
\rho_{h}=\frac{\rho C_{V} T}{c^{2}}
$$

где $\rho C_{V} T-$ плотность внутренней энергии.

Термон определяется как квазичастица, переносящая тепловую энергию.

Макроскопическая дрейфовая скорость газа термонов как сплошной среды есть

$$
v_{h}=\frac{q}{\rho C T} .
$$

Полная энергия газа термонов в среде есть сумма кинетической и потенциальной энергий [208]

$$
E_{T}=\iint_{V}\left(\rho_{T} u_{T} d u_{T}+d p_{T}\right) d V
$$

где $V$ - полный объем среды.

\section{1. Уравнение состояния (УС) газа термонов}

3.1.1. УС газа термонов в идеальном газе Два допущения делаются для газа термонов в идеальном газе:

1. Термоны связаны с молекулами газа и описываются распределением Максвелла-Больцмана.

2. Ньютоновская механика применима к газу термонов.

Давление в системе $n$ частиц с массой $m$, движущихся случайным образом в направлении $x$ со скоростью uх:

$$
P=n m u_{x}^{2}
$$

и с учетом симметрии в направлениях $x, y, z$ $\left(u_{x}^{2}=u_{y}^{2}=u_{z}^{2}=\frac{1}{3} \bar{u}^{2}\right)$ [206],

$$
P=\frac{1}{3} n m_{h} \bar{u}^{2}=\frac{1}{3} n \bar{u}^{2}\left(\frac{1}{2} \frac{m \bar{u}^{2}}{c^{2}}\right)=\frac{1}{6} \frac{n m \bar{u}^{4}}{c^{2}} .
$$

Используя функцию распределения Максвелла-Больцмана

$$
f_{M}(u)=4 \pi u^{2}\left(\frac{m}{2 \pi k_{B} T}\right)^{3 / 2} \exp \left(\frac{m u^{2}}{2 k_{B} T}\right),
$$

получаем

$$
\bar{u}^{4}=\int_{0}^{\infty} u^{4} f_{M}(u) d u=15\left(\frac{k_{B} T}{m}\right)^{2}
$$


и окончательно

$$
P=\frac{5}{3} \frac{\rho C_{V} R T^{2}}{c^{2}},
$$

где $R$ - газовая постоянная.

Таким образом, давление газа термонов в идеальном газе пропорционально квадрату температуры.

3.1.2. УС газа термонов в диэлектриках Фононы - термоны в диэлектриках. Полная энергия колебаний решетки

$$
E_{D}=E_{D 0}+E_{h}=\left(M_{0}+M_{h}\right) C_{V} T,
$$

где $E_{h}-$ энергия термомассы. Тогда давление газа термонов равно

$$
P=\frac{\gamma \rho}{c^{2}}\left(C_{V} T\right)^{2},
$$

где $\gamma$ - константа Грюнейзена.

Давление газа термонов пропорционально квадрату температуры как в идеальном газе.

Для кремния при комнатной температуре давление газа термонов около $5 \cdot 10^{-3} \mathrm{~Pa}[206]$.

3.1.3. УС газа термонов в металлах В металлах термоны присоединены к электронам. Давление газа термонов

$$
P=\frac{1}{3} n m_{h} u_{h}^{2}
$$

где $m_{h}=\varepsilon / c^{2}, \varepsilon-$ внутренняя энергия, включающая вклады электронов и решетки, $u_{h}=\sqrt{\frac{2 \varepsilon}{m}}$ скорость случайно движущихся частиц. Таким образом, давление

$$
P=\frac{2}{3} \frac{n \varepsilon^{2}}{c^{2} m} .
$$

Общее уравнение для давления газа термонов

$$
P=\frac{2}{3 m c^{2}} \int_{0}^{\infty} \varepsilon^{2} f(\varepsilon, T) Z(\varepsilon) d \varepsilon,
$$

где

$$
f(\varepsilon, T)=\frac{1}{\exp \left(\frac{\varepsilon-\varepsilon_{F}}{k_{B} T}\right)+1}
$$

— функция распределения Ферми-Дирака и

$$
Z(\varepsilon)=\frac{1}{2 \pi^{2}}\left(\frac{2 m}{\hbar^{2}}\right)^{3 / 2} \varepsilon^{1 / 2}
$$

- функция плотности состояний электронов Зоммерфельда. Ванг [206] получил следующее выражение для давления газа термонов:

$$
P=\frac{5}{12} \frac{\pi^{2} n k_{B}^{2}}{c^{2} m} T^{2} .
$$

\section{2. Уравнения движения газа термонов}

Одномерные уравнения сохранения массы и импульса

$$
\frac{\partial \rho_{h}}{\partial t}+\frac{\partial \rho_{h} u_{h}}{\partial x}=\frac{S}{c^{2}}
$$

где $S$ - источник тепла и

$$
\frac{\partial}{\partial t}\left(\rho_{h} u_{h}\right)+\frac{\partial}{\partial x}\left(u_{h} \cdot \rho_{h} u_{h}\right)+\frac{\partial P}{\partial x}+f_{h}=0 ;
$$

$f_{h}$ - сопротивление.

Уравнение неразрывности газа термонов - фактически уравнение сохранения энергии.

Течение термонов в твердом теле можно рассматривать как движение сжимаемой жидкости в пористой среде, поэтому закон Д’Арси $(K-$ проницаемость пористой среды)

$$
u=-K \frac{d P}{d x}
$$

можно использовать для оценки сопротивления термомассе $f_{h}=\beta_{h} u_{h}, \beta-$ коэффициент пропорциональности [209]

$$
\beta_{h}=\frac{e \gamma \rho^{2} C^{3} T^{2}}{c^{2} \lambda} .
$$

Эффективная сила сопротивления $f_{h}$ вводится вместо вязкого члена $\left(\mu_{h} \nabla^{2} \mathbf{u}_{h}\right)$, чтобы избежать [203]:

1) определения вязкости $\mu_{h}$ сложных материалов;

2) эффектов взаимодействия термононов с решеткой.

Течение газа термонов в твердом теле (течение фононов) вызывается градиентом давления - значит, градиентом квадрата температуры [2].

Термомасса слишком мала, чтобы допустить наблюдения в обычных условиях. Однако при сверхбыстром нагреве или чрезвычайно высоком значении теплового потока инерция термомассы приводит к эффектам в теплопроводности, которые допускают их обнаружение.

Закон сохранения импульса газа термонов можно записать как уравнение теплопроводности [206]

$$
\tau_{h}=\left(\frac{\partial q}{\partial t}+2 u_{h} \frac{\partial q}{\partial x}-u_{h}^{2} \rho C_{V} \frac{\partial T}{\partial t}\right)+\lambda \frac{\partial T}{\partial t}+q=0 .
$$

Ванг [206] разработал двухшаговую версию термомассовой теории для металлов, подвергнутых ультрабыстрому лазерному нагреву, при следующих допущениях:

1) электроны поглощают лазерную энергию и передают ее решетке;

2) рассеянием на дефектах и границах зерен пренебрегается;

3) взаимодействие электронов и фононов описывается коэффициентом связи.

По аналогии с течением сжимаемой жидкости в пористой среде поправка Бринкмана $\mu \nabla^{2} \mathbf{q}$, которая учитывает дополнительное сопротивление из-за стенок, может быть введена в уравнения движения газа термонов. Эта поправка существенна только при больших значениях числа Крудсена [207]. 
Производство энтропии при движении газа термонов обеспечивается диссипацией механической энергии

$$
\frac{d E_{h}}{\partial t}+\nabla \mathbf{J}_{E_{h}}=\mathbf{f}_{h} \cdot \mathbf{u}_{h}
$$

где $E_{h}-$ механическая энергия газа термонов, $\mathbf{J}_{E_{h}}-$ поток $E_{h}$.

Полная производная плотности энтропии записывается как $[205,210]$

$$
\frac{d S}{d t}=-\nabla \mathbf{J}_{s}+\sigma_{T M}=\frac{\mathbf{q}}{\lambda T^{2}} \cdot(\mathbf{q}+\lambda \nabla T)-\frac{\nabla \cdot \mathbf{q}}{T},
$$

где $\mathbf{J}_{s}-$ поток энтропии.

\section{3. Явление запирания теплового потока}

Газ термонов - это сжимаемая жидкость, поэтому демонстрирует свойственные сжимаемой жидкости, например воздуху, эффекты.

Один из таких эффектов - поведение газа в конвергентном сопле при числе Маха, равном единице.

Термическое число Маха определяется как

$$
M a_{h}=\frac{u_{h}}{C_{h}}
$$

где скорость звука, например, в диэлектриках $C_{h}=\sqrt{2 \gamma C_{V} T}$.

При движении сжимаемого воздуха, вызываемого градиентом давления, в конвергентном канале скорость возрастает, а давление уменьшается в направлении потока. Запирание потока происходит, когда число Маха достигает единицы; при этом появляется скачок в значениях давления.

Дрейфовая скорость газа фононов, вызываемая градиентом температуры, возрастает в направлении, противоположном градиенту температуры. Запирание теплового потока происходит, когда значение термического числа Маха достигает единицы; температура при этом испытывает скачок.

Подтверждение явления запирания теплового потока получено в экспериментах по теплопроводности в одностенных углеродных нанотрубках, подвешенных между металлическими электродами.

\section{4. Мезоскопические уравнения моментов}

Бергамаско и др. [121] разработали в рамках кинетической теории ряд моментных систем уравнений (двухмоментные и трехмоментные) с введением числа Кнудсена как отношения средней длины свободного пробега носителей тепла к характерному размеру системы. Авторы ввели также понятие „призрачного“ момента.

\section{5. Термодинамические модели}

Термодинамические модели теплопроводности выводятся из термодинамических ограничений, следующих из второго закона термодинамики [211-218].

Например, Жоу и Симелли [216] ввели новую дополнительную переменную, представляющую собой тензор второго порядка $\hat{Q}$ и записали уравнение баланса в виде

$$
\tau_{l} \dot{\mathbf{q}}+\mathbf{q}=-\lambda \nabla T+\nabla \cdot \hat{Q},
$$

где $\tau_{l}$ - время релаксации. Тензор $\hat{Q}$ предполагается симметричным и может быть расщеплен $\hat{Q}=Q \hat{I}+\hat{Q}_{s}$, где скаляр $Q$ - одна третья часть следа $\hat{Q}, \hat{Q}_{s}-$ девиаторная часть $\hat{Q}$.

Авторы вывели общее уравнение, включающее, как частные случаи уравнения Каттанео и Гюйера-Крумхансля

$$
\begin{aligned}
& \tau_{R} \mathbf{q}+\mathbf{q}+\left(\mu \nabla \mathbf{q}+\mu^{\prime} \nabla^{t} \mathbf{q}\right)-\lambda \\
& \times(1+\xi \mathbf{q} \cdot \mathbf{q}) \nabla T+l_{p}^{2}\left(\nabla^{2} \mathbf{q}+2 \nabla \nabla \cdot \mathbf{q}\right),
\end{aligned}
$$

где $\mu, \mu^{\prime}, \xi-$ материальные коэффициенты, $\nabla^{t} \mathbf{q}$ означает транспонирование $\nabla \mathbf{q}$.

Ковач и Ван [213] также ввели тензор второго порядка как внутреннюю переменную и предположили, что поток энтропии можно записать как

$$
\mathbf{J}=\hat{b} \cdot \mathbf{q}+\hat{B}: \hat{Q},
$$

где $\hat{b}-$ тензор второго порядка и $\hat{B}-$ тензор третьего порядка, называемые токовыми множителями (множителями Ниири).

Используя ограничения, следующие из второго закона термодинамики (неотрицательное производство энтропии), и исключая внутреннюю переменную, авторы вывели общее определяющее соотношение для теплового потока

$$
\begin{aligned}
& m_{1} m_{2} \partial_{t t} q+\left(m_{1} l_{1}+m_{1} k_{1}\right) \partial_{t} q \\
& -\left(m_{1} n+m_{2} k_{2}\right) \partial_{x x t} q+n k_{2} \partial_{x}^{4} q-\left(l_{1}+K\right) \partial_{x x} q \\
& +k_{1} l_{1} q=m_{2} \partial_{x t} \frac{1}{T}+k_{1} \partial_{x} \frac{1}{T}+\partial_{x}^{3} \frac{1}{T} .
\end{aligned}
$$

Выбирая материальные коэффициенты, которые можно положить равными нулю, можно получить ряд известных моделей теплопроводности, например:

- Фурье,

- Каттанео,

- баллистико-диффузионная модель,

- типа Джеффри,

- Гюйера-Крумхансля.

Роголино и др. [218] в качестве основных переменных выбрали удельную (на единицу объема) внутреннюю энергию, тепловой поток и поток теплового потока. Предполагая вид соответствующих уравнений баланca, используя энтропийные ограничения и множители 
Лагранжа-Фаркаса, авторы получили две версии обобщенного закона теплопроводности:

1) уравнение второго порядка по пространству и первого по времени в пренебрежении нелокальными эффектами;

2) уравнение четвертого порядка по пространству и второго по времени, включающее нелокальные эффекты.

\section{6. Нелокальные модели с дробными производными}

\section{1. Дробные производные}

Не существует единого определения дробной производной [219--222]. Наиболее употребительными являются определения Римана-Лиувилля и Капуто. Обе производные основаны на дробном интеграле Римана-Лиувилля, который для любого $\lambda>0$ определяется как [223,224]

$$
J_{t}^{\alpha} f(t)=\frac{1}{\Gamma(\alpha)} \int_{0}^{1}(t-\tau)^{\alpha-1} f(\tau) d \tau .
$$

Здесь $\Gamma(\alpha)=\int_{0}^{\infty} \exp (-\alpha) u^{\alpha-1} d u-$ гамма функция Эйлера.

Этот интеграл существует, если $f(t)$ локально интегрируема и при $t \rightarrow 0$ ведет себя как $O\left(t^{-v}\right)$, где $v<\alpha$.

- Дробная производная Римана-Лиувилля определяется как [225]

$$
\begin{aligned}
& \frac{\partial^{\alpha} u(x, t)}{\partial t^{\alpha}} \\
& = \begin{cases}\frac{1}{\Gamma(1-\alpha)} \int_{0}^{1} \frac{\partial u(x, s)}{\partial t}(t-s)^{-\alpha} d t, & \alpha \in(0,1) \\
\frac{\partial u(x, t)}{\partial t}, & \alpha=1 .\end{cases}
\end{aligned}
$$

- Дробная производная Капуто определяется как [225]

$$
\begin{aligned}
& \frac{\partial^{\alpha} u(x, t)}{\partial t^{\alpha}} \\
& = \begin{cases}\frac{1}{\Gamma(1-\alpha)} \int_{0}^{t} \frac{\partial u(x, s)}{\partial t}(t-s)^{-\alpha} d t, & \alpha \in(0,1) \\
\frac{\partial u(x, t)}{\partial t}, & \alpha=1 .\end{cases}
\end{aligned}
$$

Известен ряд определений дробных производных помимо Римана-Лиувилля и Капуто (Грюнфельд-Летникова, Рисза, Вейля, Марчо, Капуто-Фабрицио, Янга, Чена, Хе. Жао, Кая-Янга и некоторые другие), которые не эквивалентны [226].

\section{2. Дифференциальные уравнения с дробными производными}

Обычно фрактальную среду нельзя рассматривать как сплошную. Использование пространств нецелой размерности [227] необходимо для описания фрактальной среды с помощью континуальных моделей [228]. Уравнения с дробными производными [229] (первыми дробное исчисление использовали Абель и Лиувилль) нелокальны (т.е. могут включать эффекты памяти и пространственные корреляции) и могут описывать аномальную диффузию (как субдиффузию, так и супердиффузию) и аномальную теплопроводность [230] — например, теплопроводность в пористой среде описывается моделью супердиффузии [231]).

Начальные условия для дробной производной Капуто можно сформулировать в терминах начальных условий для целочисленных производных. Нулевые начальные условия для производных Римана-Лиувилля, Капуто и Грюнфельда-Летникова совпадают [232].

\section{3. Дробная модель Фурье}

Денг и Ге [233] изучали теплоперенос в фрактальной среде, используя дробное уравнение Гельмгольца

$$
\frac{\partial^{\alpha} T(x, y)}{\partial x^{2 \alpha}}+\frac{\partial^{2 \beta} T(x, y)}{\partial y^{2 \beta}}+k^{2} T(x, y)=f(x, y),
$$

где $0<\alpha \leq 1,0<\beta \leq 1$.

Хе и Лиу [234] использовали дробную версию закона Фурье

$$
\lambda^{2 \alpha} \frac{\partial^{\alpha} T}{\partial x^{\alpha}}=q
$$

для изучения теплообмена в системе коконов шелковичного червя.

Подобный подход использовали Бейбалаев и др. для исследования теплопереноса в фрактальной среде и для изучения промерзания грунта $[235,236]$.

Хе и др. [237] использовали стационарное

$$
\frac{\partial^{\alpha}}{\partial x^{\alpha}}\left(\lambda \frac{\partial^{\alpha} T}{\partial x^{\alpha}}\right)=0
$$

а Ванг и др. [238] нестационарное уравнение

$$
\frac{\partial T}{\partial t}+\frac{\partial^{\alpha}}{\partial x^{\alpha}}\left(\lambda \frac{\partial^{\alpha} T}{\partial x^{\alpha}}\right)=0
$$

для исследования теплопереноса в рамках фрактальной модели меха белого медведя.

Мейланов и Шабанова [239] решали одномерные задачи для дробного уравнения

$$
\frac{\partial^{\alpha} T}{\partial t^{\alpha}}=\lambda \frac{\partial^{\beta} T}{\partial t^{\beta}}
$$

Воллер и др. [240] использовали дробную по времени, а Мейланов и др. [241] - дробную по времени и пространству модель для решения задачи Стефана. Воллер и др. рассмотрели случаи как резкой, так и диффузной границы между жидкой и твердой фазами.

Сироцюк и др. использовали дробное по времени уравнение Фурье для расчета теплопереноса неоднородной полубесконечной балке [242]. 


\section{4. Дробная модель Пеннеса}

Обобщение с помощью дробной производной Капуто уравнения Пеннеса (2)

$$
\rho c \frac{\partial^{\alpha} T}{\partial t^{\alpha}}=\nabla \cdot \lambda \nabla T+c_{b} \omega_{b}\left(T_{a}-T\right)+\dot{g}_{m e t}+Q^{e x t},
$$

было использовано Дамором и др. [243] для исследования гипертермии и аномальной теплопроводности в тканях кожи при постоянном и синусоидальном нагреве поверхности $[244,245]$ и Эззатом и др. [246] для расчета нестационарного теплопереноса в коже при мгновенном нагреве поверхности.

Как отметили Феррас и др. [247], уравнение (7) некорректно с точки зрения размерности, и необходимо либо переопределить коэффициенты в классическом уравнении, либо ввести множитель $\tau^{1-\alpha}$ для получения „новой“ теплопроводности.

Сингх и др. [248] для анализа поля температуры в тканях при гипертермии применяли дробное по времени и пространству уравнение

$$
\rho C \frac{\partial^{\beta}}{\partial t^{\beta}} T=\lambda \frac{\partial^{\alpha}}{\partial x^{\alpha}}+Q_{p}, \quad 0<\beta \leq 1<\alpha \leq 2 .
$$

\section{5. Дробная модель Зингалеса}

Зингалес [249] (см. также [250]) рассмотрел две компоненты теплопереноса в твердых телах в покое:

1) „близкий“ теплоперенос, описываемый обычным законом Фурье;

2) теплообмен между удаленными элементарными объемами, расположенными в точках $\mathbf{x}$ и $\mathbf{y}$, который пропорционален

- произведению взаимодействующих масс;

- разности температур $T(\mathbf{x})-T(\mathbf{y})$;

- убывающей функции расстояния $g(\|\mathbf{x}-\mathbf{y}\|)$.

Автор предположил, что функция $g$ убывает как степенная функция расстояния

$$
g(\|\mathbf{x}-\mathbf{y}\|)=\frac{1}{d_{n}(\bar{\alpha})} \cdot \frac{1}{\|\mathbf{x}-\mathbf{y}\|^{n+\alpha}},
$$

где $d_{n}(\bar{\alpha})$ - нормализующий коэффициент, связанный с убывающей экспонентой $\alpha$ и размерностью топологического пространства тела $n$.

Окончательно уравнение сохранения энергии записывается в виде

$$
\rho C \frac{\partial T}{\partial t}=-\nabla \mathbf{q}+\rho^{2} \lambda_{\alpha} D_{x}^{\alpha} T,
$$

где $D_{x}^{\alpha}$ - дробная производная Марчо порядка $\alpha$ определяется как

$$
D_{x}^{\alpha} T=\frac{1}{d_{n}(\bar{\alpha})} \int_{V_{y}}^{\square} \frac{T(\mathbf{x})-T(\mathbf{y})}{\|\mathbf{x}-\mathbf{y}\|^{n+\alpha}} d V_{y} .
$$

\section{6. Дробные модели Каттанео и SPL}

Иногда дробную версию уравнения Каттанео называют „нелокальным“ уравнением Каттанео-Вернотта, что отражает свойства дробных производных [251].

Лиу и др. [252] использовали модификацию Христова модели Каттанео для разработки дробного уравнения с применением пространственно-дробной производной Рисза.

Дробная по времени SPL-модель биологического теплообмена сформулирована в работе [253]

$$
\begin{aligned}
& \rho c\left(\frac{\partial^{\alpha} T}{\partial t^{\alpha}}+\tau \frac{\partial^{1+\alpha} T}{\partial t^{1+\alpha}}\right) \\
& =\nabla \cdot \lambda \nabla T+c_{b} \omega_{b}\left(T_{a}-T\right)+\dot{g}_{m e t}+Q^{e x t} .
\end{aligned}
$$

Вычисления показывают, что дробная SPL-модель дает то же распределение температуры, что и DPLмодель [253].

Дробная по пространству SPL-модель сформулирована для одномерного случая Кумаром и др. [254]

$$
\rho c \frac{\partial T}{\partial t}=-\frac{\partial^{\alpha} q}{\partial x^{\alpha}}+c_{b} \omega_{b}\left(T_{b}-T\right)+Q^{e x t},
$$

где

$$
q(x, t)+\tau \frac{\partial q(x, t)}{\partial x}=-\lambda \nabla T,
$$

и для $m-1<\alpha<m$

$$
\frac{\partial^{\alpha} u(x, t)}{\partial x^{\alpha}}=\frac{1}{\Gamma(m-\alpha)} \int \frac{\partial^{m} u(x, s)}{\partial s^{m}}(x-s)^{m-\alpha-1} d s,
$$

здесь $\Gamma(z)$ - гамма-функция.

Джианг и Кви [255] вывели дробную модель тепловой волны, изменив соотношение Каттанео

$$
\frac{\tau^{\alpha}}{\alpha !} D_{t}^{\alpha} \mathbf{q}+\mathbf{q}=-\lambda \nabla T,
$$

где $D_{t}^{\alpha}-$ модифицированная производная Римана-Лиувилля порядка $\alpha$.

Кви и др. [256] изучали лазерный нагрев, обобщив соотношение Каттанео как

$$
\tau^{p} D_{t}^{p} \mathbf{q}+\mathbf{q}=-\lambda \nabla T,
$$

где $D_{t}^{p}-$ производная Капуто порядка $p$; множитель $\tau^{p}$ введен для коррекции размерности.

Ху и др. предложили дробное уравнение Каттанео, используя две производные Капуто разного порядка

$$
\begin{gathered}
\frac{\partial^{\beta-1} \mathbf{q}}{\partial t^{\beta-1}}+\tau \frac{\partial^{\alpha-1} \mathbf{q}}{\partial t^{\alpha-1}}+\mathbf{q}=-\lambda \nabla T, \quad 0<\beta \leq \alpha \leq 2, \\
\frac{\tau^{\alpha}}{\Gamma(1+\alpha)} \frac{\partial^{\alpha} \mathbf{q}}{\partial t^{\alpha}}+=-\lambda \nabla T, \quad 0<\alpha \leq 1 .
\end{gathered}
$$

Мишра и Рай [257] использовали дробную SPL-модель для анализа теплопереноса в тонких пленках. 
Мороз и Масловская [258] использовали дробную по пространству SPL-модель для моделирования теплопроводности в сегнетоэлектриках (триглицинсульфат).

Христов [259] разработал дробное по пространству уравнение нестационарного теплопереноса с демпфирующим членом, описываемым с помощью производной Капуто-Фабрицио [260], которая модифицирует производную Капуто

$$
D_{t}^{\alpha} f(t)=\frac{M(\alpha)}{1-\alpha} \int_{0}^{t} \exp \left[-\frac{\alpha(t-s)}{1-\alpha}\right] \frac{d f(t)}{d t} d s,
$$

где $M(\alpha)$ - нормализующая функция, такая, что $M(0)=M(1)=1$.

Численное решение этой задачи рассмотрели Алкахтани и Атангана [261].

Танг и др. [262] (см. также [263]) для дробного теплопереноса ввели новую дробную производную без сингулярного ядра как модификацию производной Римана-Лиувилля

$$
D_{\alpha^{+}}^{(v)} T=\frac{\mathscr{R}(v)}{(1-v)} \frac{d}{d x} \int_{a}^{x} \exp \left[-\frac{v}{1-v}\left(x-x^{\prime}\right)\right] T d x^{\prime},
$$

где $\mathscr{R}(v)$ - нормализующая функция.

\section{7. Дробная DPL-модель}

Джи $[264,265]$ использовал следующую форму дробной по времени DPL-модели $\left({ }_{0}^{C} D_{t}^{\alpha}-\right.$ дробная производная Капуто

$$
\begin{array}{r}
\rho C\left(\frac{\partial T}{\partial t}+\frac{\tau_{q}^{\alpha}}{\Gamma(1-\alpha)}{ }_{0}^{C} D_{t}^{1+\alpha} T\right) \\
\quad=\lambda\left(T+\frac{\tau_{T}^{\alpha}}{\Gamma(1-\alpha)}{ }_{0}^{C} D_{t}^{\alpha} T\right)
\end{array}
$$

для исследования теплообмена в тонких пленках.

$\mathrm{Xu}$ и др. [266] изучали биологический теплообмен, используя уравнение с производными Капуто порядков $\alpha$ и $\beta$

$$
\mathbf{q}+\tau_{q}^{\alpha} \frac{\partial^{\alpha} \mathbf{q}}{\partial t^{\alpha}}=-\lambda\left(\nabla T+\tau_{T}^{\beta} \frac{\partial^{\beta}}{\partial t^{\beta}} \nabla T\right) .
$$

Авторы заменили релаксационные времена DPLмодели $\tau_{q}$ и $\tau_{T}$ на $\tau_{q}^{\alpha}$ и $\tau_{T}^{\beta}$ для сохранения размерности.

Лиу и др. [267] использовали конвективную производную, введенную Христовым [123], и производную Капуто порядка $\alpha$ для формулировки модели

$$
\begin{gathered}
\tau_{q}\left[\frac{\partial^{\alpha} \mathbf{q}}{\partial t^{\alpha}}+\mathbf{v} \cdot \nabla \mathbf{q}+\mathbf{q} \cdot \nabla \mathbf{v}+(\nabla \cdot \mathbf{v}) \mathbf{q}\right]+\mathbf{q} \\
=-\lambda \nabla\left(1+\tau_{T} \frac{\partial^{\alpha}}{\partial t^{\alpha}}\right) T .
\end{gathered}
$$

\section{8. Дробная TPL-модель}

Ахбарзадех и др. [184] обобщили определяющее соотношение TPL-модели и сохранили члены до $2 \alpha_{F}$-порядка для $\tau_{q}$ и до $\alpha_{F}$-порядка для $\tau_{T}$ и $\tau_{v}$ и получили

$$
\begin{aligned}
& \left(1+\frac{\tau_{q}^{\alpha_{F}}}{\alpha_{F} !} \frac{\partial^{\alpha_{F}}}{\partial t^{\alpha_{F}}}+\frac{\tau_{q}^{2 \alpha_{F}}}{\left(2 \alpha_{F}\right) !} \frac{\partial^{2 \alpha_{F}}}{\partial t^{2 \alpha_{F}}}\right) \mathbf{q} \\
& =\left[\left(\lambda+\frac{\lambda \cdot \tau_{q}^{\alpha_{F}}}{\left(\alpha_{F}\right) !} \frac{\partial^{\alpha_{F}-1}}{\partial t^{\alpha_{F}-1}}+\frac{\lambda \tau_{q}^{\alpha_{F}}}{\left(\alpha_{F}\right) !} \frac{\partial^{\alpha_{F}}}{\partial t^{\alpha_{F}}}\right) \nabla T+\lambda \cdot \nabla v\right] .
\end{aligned}
$$

Дробная TPL-модель, как и обычная TPL, используется для решения задач термоупругости [268,269].

\section{Заключение}

В обзоре сделана попытка охватить все многообразие моделей, предназначенных для анализа теплопереноса за пределами закона Фурье.

Наиболее перспективными представляются термодинамические и дробные модели, для исследования теплопереноса в диэлектриках привлекательна модель релаксонов.

Как показывает анализ литературы, для исследования теплообмена в биологических тканях чаще всего применяется DPL-модель.

\section{Приложение. Некоторые точные решения}

Точные аналитические решения способствуют изучению процессов теплообмена и служат в качестве эталонов при разработке численных методов.

Фан и Ванг опубликовали обширный обзор точных решений задач биологического теплообмена, включая уравнение Пеннеса, модель тепловой волны и DPLмодели [270].

Саркар и др. [271] рассмотрели стационарный теплоперенос в многослойной ткани кожи, используя уравнение Пеннеса и модели с задержкой для случаев задания температуры или теплового потока.

Модели с задержкой Барлетта и Заечини [272] анализировали теплоперенос в бесконечно широкой полосе с заданным тепловым потоком, используя уравнение Каттанео. Подобная задача в цилиндрических координатах решалась Саердолином и др. [273].

Ахмадикиа и др. [274, 275] и Кунду и Деванжи [276] использовали модель тепловой волны и уравнение Пеннеса для моделирования воздействия на ткань кожи постоянного и импульсного нагрева.

Аль-Хаири и др. [277] исследовали лазерное воздействие (постоянное, мгновенное или экспоненциальное) на движущуюся полубесконечную среду в рамках модели Каттанео. 
DPL-модель использована Аскаризадех и Ахмадикиа $[278,279]$ и Лином [280] для исследования нестационарного нагрева тканей кожи.

Акхаири [281] решал уравнения DPL-модели в однородном материале, используя функцию Грина, Дай и Нассар [282] использовали исходную форму DPL-модели без разложения Тейлора.

Кулиш и Новожилов [141] вывели, используя преобразование Лапласа, интегральное уравнение, связывающее температуру с ее градиентом.

Жанг [148] использовал метод „изготовленных решений“, который основан на угадывании возможного решения и определения соответствующих граничных и начальных условий.

Жуковский [283] (см. также [284]) вывел точное решение уравнения Гюйера-Крумхансля, используя операторный метод, и обнаружил, что в некоторых режимах решение может нарушать принцип максимума. Однако это заключение не подтверждается в недавней работе Ковача [285]. Начальные и граничные условия соответствовали лазерному эксперименту. Решение находилось в двух интервалах: $0<t<\tau_{\Delta}$ и $t>\tau_{\Delta},\left(\tau_{\Delta}-\right.$ длительность лазерного импульса).

Дробные модели Дейикайа и Кимаз [286] использовали метод обобщенного дифферанцального преобразования для решения уравнения диффузии с производной Капуто.

Повстенко получил точные решения для теплопроводности в двух сопряженных полулиниях [287], полубесконечном композитном материале [288], в среде со сферическими включениями [289].

Джуний и Мингу [290] получили решение задачи Стефана, Янг и др. [291], решая одномерную нестационарную задачу для уравнения с локальной дробной производной.

Каземи и Ерджии [292] изучали дробное уравнение диффузии.

Гош и др. [293] вывели решение линейного дробного уравнения с производной Джумари в терминах функций Миттаг-Лефлера.

\section{Конфликт интересов}

Автор заявляет, что у него нет конфликта интересов.

\section{Список литературы}

[1] J. Lebon, H. Machraft, M. Grmela, C. Debois. Proc. R. Soc. A, 467, 3241 (2011).

[2] B.J. Cao, Z.Y. Guo. Appl. Phys., 102, 053503 (2007).

[3] M. Chester. Phys. Rev., 131, 2013 (1963).

[4] R.F. Hu, B.Y. Cao. Sci. Chine Ser. E: Technol. Sci., 52, 1786 (2009).

[5] J.B. Brown, D.Y. Chung, P.W. Matthews. Phys. Lett. 21, 241 (1966).

[6] K.K. Tamma, X.J. Zhou. Thermal Stresses, 21, 405 (1998).
[7] C.C. Ackerman, W.C. Overton. Phys. Rev. Lett., 22, 764 (1969).

[8] T.F. McNelly, S.J. Rogers, D.J. Channin, R. Rollefson, W.M. Goubau, G.E. Schmidt, J.A. Krumhansl, R.O. Pohl. Phys. Rev. Lett. 24, 100 (1970).

[9] H. Jackson, C.I. Walker. Phys. Rev. B, 3, 1428 (1971).

[10] R. Kovacs, P. Van. arXiv: 1708.09770 [cond-mat.stat-mech], (2017)

[11] V. Narayanamurti, R.C. Dynes. Phys. Rev. Lett., 28, 1461 (1972).

[12] M. Simoncelli, N. Marzari, A. Cepellotti. Phys. Rev. X, 10, 011019 (2020).

[13] S. Huberman, R.A. Duncan, K. Chen, B. Song, V. Chiloyan, Z. Ding, A.A. Maznev, G. Chen, K.A. Nelson. Science, 364, 375 (2019).

[14] D. Sands. In Heat Transfer - Engineering Applications, (Edit.V. Vikhrenko), (InTech, 2011)

[15] A. Bannerjee, A.A. Ogale, C. Das, K. Mitra, C. Subranian. Heat Transfer Eng., 26, 41 (2005).

[16] H.L. Lee, W.L. Chen, W.J. Chang, E.J. Wei, Y.C. Yang. Appl. Themal Eng. 52, 275 (2013).

[17] D.Y. Ho, M.Y. Wen, B.C. Chen, Y.H. Tsai. J. Nanosci. Nanotechnol., 14, 1 (2014).

[18] Y.D. Mao, M.T. Xu. Sci. China Tech. Sci., 58, 1 (2015).

[19] Z. Shomali, A. Abbassi. Int. J. Thermal Sci., 83, 56 (2014).

[20] A.M. Mullis. Int. J. Heat Mass Transfer ., 40, 4085 (1997).

[21] R. Hilfer. Chem. Phys., 284, 399 (2002).

[22] P. Van, A. Berezovski, T. Fulop, G. Grof, R. Kovacs, A. Lovas, J. Verhas. arXiv: 1704.00341 [cond-mat.stat-mech], (2017)

[23] D.G. Cahill, W.K. Ford, K.E. Goodson, G.D. Mahan, A. Majumdar, H.J. Maris, R. Merlin, S.R. Phillpot. J. Appl. Phys., 93, 793 (2003).

[24] Z.M. Zhang. Nano/Microscale Heat Transfer. (McGraw-Hill, N.Y., 2007).

[25] D.G. Cahill, P.V. Braun, G. Chen, D.R. Clark, S. Fan, K.E. Goodson, P. Keblinski, W.P. King, G.D. Mahan, A. Majumdar, H.J. Maris, S.R. Phillpot, E. Pop, L. Shi. Appl. Phys. Rev., 1, 011305 (2014).

[26] В.И. Хвесюк, А.С. Скрябин. ТВТ, 55, 447 (2017).

[27] S. Sinba, K.E. Goodson. J. Microel. J., 37, 1148 (2006).

[28] B. Vermeersch, G. De May. Analog. Integr. Circ. Sig. Process., 55, 197 (2008).

[29] K. Raleva, D. Vasileska, S.M. Goodnick, M. Nedjalkov. IEEE Trans. Electron Devices 55, 1306 (2008).

[30] T. Raszkowski, A. Samson. Comput. Sci., 18, 71 (2017).

[31] P.G. Sverdrup, S. Sinha, M. Asheghi, S. Uma, K.E. Goodson. Appl. Phys. Lett. 78, 3331 (2001).

[32] P.G. Sverdrup, Y. Sungtaek, K.E. Goodson. Trans. ASME, 123, 130 (2001).

[33] E. Pop, S. Sinha, K.E. Goodson. Proc. IEEE, 94, 1587 (2006).

[34] E. Pop, K.E. Goodson. J. Electron. Packag., 128, 102 (2006).

[35] M.G. Kanatdzikis. Chem. Mater., 22, 648 (2009).

[36] F. Vazqurz, P. Van, R. Kovacs. Entropy, 22, 167 (2020).

[37] П.П. Волосевич, Н.В. Змитренко, Е.И. Леванов, Е.В. Северина. Матем. Модел., 20, 57 (2008).

[38] K.A. Velizhanin, C.C. Chien, Y. Dubi, M. Zwolak. arXiv: 1101.002 [cond-mat.mes-hall], (2011). 
[39] K.M. Hoogeboom-Pot, J.N. Hernandez-Charpak, X. Gu, T.D. Frazer, E.H. Anderson, W. Chao, R.W. Falcone, R. Yang, M.M. Murnane, H.C. Kapteyn, D. Nardi. PNAS, 112, 4846 (2015).

[40] A. Grassman, F. Peters. Heat Mass Transfer, 35, 289 (1999).

[41] J. Ordonez-Miranda, J.J. Alvarado-Gill. Granular Mater., 12, 569 (2010).

[42] F.X. Alvarez, V.A. Cimmelli, A. Sellitto. Nanosc. Systems, 1, 112 (2012).

[43] K.C. Liu, P.J. Chen. J. Thermophys. Heat Transfer, 22, 775 (2008).

[44] A.I. Zhmakin. Fundamentals of Cryobiology. Physical phenomena and mathematical models. (Springer Series „Biological and Medical Physics“), (Springer, Berlin, 2009).

[45] H. Ahmadikia, A. Moradi. Heat Mass Transfer, 48, 1559 (2012).

[46] D.F. Stranges, R.E. Khayat, B. Albaalbaki. Int. J. Thermal Sci., 74, 14 (2013).

[47] D.F. Singh, S. Kumar. Math. Model. Anal., 20, 443 (2015).

[48] C.B. Sobban, S. Thomas, G.P. Peterson. Adv. Nanomater., 2, 41 (2017).

[49] A.I. Zhmakin. Handbook of Thermal Science and Engineering, Edit: F. Kulacki, (Springer, 2018).

[50] R.B. Wilson, D.J. Cahill. Appl. Phys. Lett., 107, 203112 (2015).

[51] R.R. Alfano, S.G. Demos, S.K. Guyen. Ann. N. Y. Acad. Sci., 820, 248 (1997).

[52] A. Obana, Y. Gohto. Las. Surg. Med., 30, 170 (2002).

[53] M. Panjehpour, A. Wilke, D.J. Frazier, B.F. Overholt. Proc. SPIE, 1427, 307 (1991).

[54] F.H. Loesel, E.P. Fisher, H. Suhan, J.F. Bille. Appl. Phys. B, 66, 121 (1998).

[55] S.W. Jeong, H. Liu, W.R. Chen. Proc. SPIE, 5068, 216 (2003).

[56] K.C. Liu. Int. J. Thermal Sci., 47, 507 (2008).

[57] X. Li, Y. Zhong, J. Smith, C. Gu. Bioengin., 8, 71 (2017).

[58] Y. Hou, Z. Sun, W. Raw, J. Liu. Nanomed. Nanotechnol. Biol. Med., 14, 493 (2018).

[59] D.J. Tzou, W. Dai. Int. J. Heat Mass Transfer, 52, 1206 (2009).

[60] B. Rethfeld, A. Kaiser, M. Vicanck, G. Simon. Phys. Rev. B, 65, 214303 (2002).

[61] Д.С. Поляков, Е.Б. Яковлев. Квантовая электроника, $\mathbf{4 5}$ 917 (2015).

[62] S.V. Anisimov, B.L. Kapeliovich, T.L. Perelman. Sov. Phys. JETP, 39, 375 (1974).

[63] T.Q. Qiu, C.L. Tien. Int. J. Heat Mass Transfer, 37, 2789 (1994).

[64] J. Liu. Forsch. Ingenieur., 66, 1 (2000).

[65] D.T.W. Lin. Int. J. Sci. Eng., 1, 17 (2011).

[66] T. Nakano, G. Kikugawa, T.J. Ohara. Heat Transfer, 135, 661301 (2013).

67] D.J. Cahill. Rev. Sci. Instr., 61, 802 (1990).

[68] C.C. Williams, H.K. Wickramasinghe. Appl. Phys. Lett., 49, 1587 (1986).

[69] D. Poulikakos, S. Arcidiacono, S. Maruyama. Microsc. Thermophys. Eng., 7, 181 (2003).

[70] W.J. Kaminski. Heat Transfer, 112, 555560 (1990).

[71] S.L. Sobolev. Sov. Phys. Usp., 34, 217 (1991).

[72] M.N. Ozisik, D.Y. Tzou. J. Heat Transfer, 116, 526535 (1994).
[73] M. Janicki, G. DeMay, M. Zubert, A. Napieralski. 30th SEMI-THERM Symposium, 202-206, (2014).

[74] R.E. Khayat, J. deBruyn, M. Niknami, D.F. Stranges, R.M.H. Khorasani. Int. J. Thermal Sci., 997, 163 (2015).

[75] K. Mitra, S. Kumar, A. Vedavarz, M.K. Moallemi. J. Heat Transfer, 117, 568 (1995).

[76] H. Herwig, K. Beckert. Heat Mass Transfer, 36, 387 (2000).

[77] W. Roetzel, N. Putra, S.K. Das. Int. J. Thermal Sci., 42, 541 (2003).

[78] P.J. Antaki. J. Heat Transfer, 127, 189 (2005).

[79] F. Xu, K.A. Seen, T. Lu. J. Int. J. Heat Mass Transfer, 51, 2237 (2008).

[80] Y. Rabin, P.S. Steif. J. Appl. Mech., 65, 328 (1998).

[81] Y. Rabin, P.S. Steif. Int. J. Solids Struct. 37, 2363 (2000).

[82] Z.S. Deng, J. Lin. J. Thermal Stresses, 26, 779 (2003).

[83] X. Shi, A.K. Datta, S. Mukharjee. J. Thermal Stresses, 22, 275 (1999).

[84] Z.Z. Hua, H.Y. Xu, G.Y. Zhou, J.F. Liu, H. Huang, W.X. Ding. Sci. in China, Ser. E., 44, 159 (2001).

[85] X. Shi, A.K. Datta. J. Biomech. Eng., 120, 720 (1998).

[86] Q. Liu, P. Jiang, H. Xiang. Prog. Nat. Sci., 18, 999 (2008).

[87] T.H. Yu, J. Liu, Y.X. Zhou. J. Thermal Stresses, 27, 1089 (2004).

[88] I.A. Lubashevsky, V.V. Gaychuk, B.Y. Datsko. arXiv:condmat/020105/v1 [cond-mat.soft], (2002).

[89] N. Afrin, Y. Zhang, J.K. Chen. Int. J. Heat Mass Transfer, 54, 2419 (2011).

[90] H.H. Pennes. J. Appl. Physiol., 1, 93 (1948); reprinted: Ibid, 85, 5 (1998).

[91] J. Crezee, J. Lagendjik. J. W. Phys. Med. Biol., 37, 1321 (1992).

[92] A. Lakssass, E. Kengne, H. Semmaoui. Natural Sci., 2, 1375 (2010).

[93] R. Livi, S. Lepri. Nature, 421, 327 (2003).

[94] A. Sellito, F.X. Alvarez. Nanosc. Systems, 1, 38 (2012).

[95] M. Pumarol, M.C. Rosamond, P.D. Tovee, M.C. Petty, D. Zeze, V.I. Falko, O.V. Kolosov. Nano Lett., 12, 2906 (2012).

[96] A.K. Majee, Z. Aksamija. Phys. Rev. B, 93, 235423 (2016).

[97] A. Henry, G. Chen. Phys. Rev. Lett., 101, 235502 (2008).

[98] A. Henry, G. Chen. Phys. Rev. B, 79, 144305 (2009).

[99] A. Jou, A. Sellitto, F.X. Alvarez. Proc. R. Soc. A, 467, 2520 (2011).

[100] P. Grassberger, L. Yang. arXiv; [cond-mat/020424], (2002).

[101] C.W. Chang, D. Okawa, H. Garcia, A. Majumdar, A. Zettl. Phys. Rev. Lett., 101, 075903 (2008).

[102] А.В. Елецкий. УФН, 179, 225 (2009).

[103] A.J. Majumdar. Heat Transfer, 115, 7 (1993).

[104] E.M. Moares. Heat Conduction - Basic Research, Edit:V. Vikrenko, (InTech, 2011).

[105] K. Takahashi. JSME Int. J. Ser. A, 40, 99 (1997).

[106] M.E. Gurtin, A.C. Pipkin. Arch. Rat. Mech. Analysis, 31, 113 (1969).

[107] D.D. Joseph, L. Presiosi. Rev. Mod. Phys., 61, 41 (1989).

[108] J. Hristov. Thermal Sci., 17, 733 (2013).

[109] S. Rukolaine, A. Samsonov. Phys. Rev. E, 88, 06211612 (2013).

[110] A.H. Akbarzadeh, D. Pasini. Int. J. Heat Mass Transfer, 75, 656667 (2014).

[111] K.C. Liu. Int. J. Heat Mass Transfer, 81, 347 (2015).

[112] D.D. Joseph, L. Presiosi. Rev. Mod. Phys., 62, 375 (1990).

[113] S.I. Serdyukov. Theor, Found, Chem, Eng., 47, 122 (2013). 
[114] D. Jou, J. Casas-Vazouez. Physica A, 163, 47 (1990).

[115] L. Cheng, M. Xu, L. Wang. Int. J. Heat Mass Transfer, 51, 6018 (2008).

[116] S.N. Li, B.Y. Cao. Int. J. Heat Mass Transfer, 98, 824 (2016).

[117] M. Ciesielski, M. Duda, B. Mochnacki. J. Appl. Math. Comput. Mech., 15, 33 (2016).

[118] J.H. Choi, S.H. Yoon, S.G. Park, S.H. Choi. J. Korean Soc. Marine Eng., 40, 389 (2016).

[119] J.I. Frankel, B. Vick, M.N. Ozisik. J. Appl. Phys., 58, 3340 (1985).

[120] B.D. Nie, B.Y. Cao. Int. J. Heat Mass Transfer, 135, 974 (2019).

[121] L. Bergamasco, M. Alberhini, M. Fasano, A. Cardellini, E. Chiavazzo, P. Asinari. Entropy, 20, 126 (2018).

[122] C.I. Christov, P.M. Jordan. Phys. Rev. Lett., 94, 154301 (2005).

[123] C.I. Christov. Mech. Res. Comm., 36, 481 (2009).

[124] R.E. Khayat, M. Ostoja-Starzewski. Discrete Contin. Dynam. Syst., Series B, 15, 991 (2011).

[125] A. Barletta, E. Zanchini. Int. J. Heat Mass Transfer, 40, 1007 (1997).

[126] A. Barletta, E. Zanchini. Phys. Rev. B, 55, 14208 (1997).

[127] E. Zanchini. Phys. Rev. B, 60, 991 (1999).

[128] J.A. Conejero, A. Peris, M. Trujillo. Int. J. Bifurc. Chaos, 20, 2943 (2010).

[129] D. Jou, J. Casa-Vazquez, G. Lebon. Rep. Prog. Phys., 51, 1105 (1988).

[130] D. Jou, J. Casa-Vazquez, G. Lebon. Rep. Prog. Phys., 62, 1035 (1999).

[131] S.N. Li, B.Y. Cao. Entrophy, 18, 391 (2016).

[132] X. Liu, Y. Zhu, F. Zhang, X.F. Gong. Chin. Phys. B, 22, 024301 (2013).

[133] A. Salazar. Ur. J. Phys., 27, 1349 (2006).

[134] D.Y. Tzou. Int. J. Heat Mass Transfer, 36, 1845 (1993).

[135] D.J. Tzou. Int. J. Heat Mass Transfer, 38, 3231 (1995).

[136] D.Y. Tzou. Macro- to Microscale Heat Transfer: The Lagging Behavior, 2nd ed. (Wiley, N.Y., 2015).

[137] F. Xu, T. Lu. J. Adv. Appl. Math., 43, 147 (2009).

[138] W. Dai, N. Nassar. Num. Heat Transfer, 127, 243 (2000).

[139] K.C. Liu, H.T. Chen. Int. J. Thermal Sci., 49, 1138 (2010).

[140] Y. Zhang. Int. J. Heat Mass Transfer, 52, 4829 (2009).

[141] V.V. Kulish, V.B. Novozhilov. J. Heat Transfer, 126, 805 (2004).

[142] J. Ordonez-Miranda, J. Alvarado-Gill. Nanosc. Res. Lett., 6, 327 (2011).

[143] M. Xu, J. Guo, L. Wang, L. Cheng. Int. J. Heat Sci., 50, 825 (2011).

[144] M.I. Kaganov, I.M. Lifshitz, M.V. Tanatarov. Sov. Phys. JETP, 4, 173 (1957).

[145] S.I. Anisimov, B.L. Kapeliovich, T.L. Perelman. Sov. Phys. JETP, 39, 375 (1974).

[146] T.Q. Qiu, C.L. Tien. J. Heat Transfer, 115, 835 (1993).

[147] D.Y. Tzou. Int. J. Heat Transfer, 38, 3231 (1995).

[148] Y. Zhang. Int. J. Heat Mass Transfer, 52, 4829 (2009).

[149] D.Y. Tzou, W. Dai. Int. J. Heat Mass Transfer, 52, 1206 (2009).

[150] K.C. Liu. Int. J. Heat Mass Transfer, 54, 2829 (2011).

[151] K.C. Liu, P.J. Cheng, J.C. Wang. Int. J. Eng. Technol., 6, 132 (2014).

[152] L. Wang, M. Xu, X. Zhou. Int. J. Heat Mass Transfer, 44, $1650(2001)$

[153] L. Wang, M. Xu. Int. J. Heat Mass Transfer, 45, 1165 (2002).
[154] K.C. Liu, P.J. Cheng, Y.N. Wang. Thermal Sci., 15, S61 (2011).

[155] J. Escolano, F. Rodriguez, M.A. Castro, F. Vives, J.A. Martin. Math. Comput. Model., 54, 1841 (2011).

[156] S.A. Rukolaine. Int. J. Heat Mass Transfer, 78, 58 (2014).

[157] S.A. Rukolaine. Int. J. Heat Mass Transfer, 113, 83 (2017).

[158] R. Quintanilla, R. Racke. Int. J. Heat Mass Transfer, 49, 1209 (2006).

[159] R. Quintanilla. J. Non-Equilib. Thermodyn., 27, 217 (2001).

[160] M. Fabrizio, B. Lazzari. Int. J. Heat Mass Transfer, 74, 484 (2014).

[161] M. Xu. J. Heat Transfer, 133, 041401 (2011).

[162] J. Zhou, Y. Zhang. Comput. Biol. Med., 39, 288 (2009).

[163] M. Jaunich, S. Raje, K. Kim, K. Mitra, Z. Guo. Int. J. Heat Mas Transfer, 51, 5511 (2008).

[164] N. Afrin, J. Zhou, Y. Zhang, D.Y. Tzou, J.K. Chen. Numer. Heat Transfer, Part A, 61, 483 (2012).

[165] H. Ahmadikia, A. Moradi, R. Fazlali, A. Parsa. J. Mechan. Sci. Technol., 26, 1937 (2012).

[166] N. Sahoo, S. Ghosh, A. Narasimhan, S.K. Das. Int. J. Thermal Sci., 76, 208 (2014).

[167] K.C. Liu, J.C. Wang. Int. J. Heat Mass Transfer, 70, 621 (2014).

[168] H.Z. Poor, H. Moosavi, A. Moradi, H.G. Menghari, M. Parastarfeizabadi. Int. J. Mech. Syst. Eng., 4, 33 (2014).

[169] P. Hooshomand, A. Moradi, B. Khezri. Int. J. Thermal Sci., 90, 214 (2015).

[170] S. Kumar, A. Srivastava. Int. J. Heat Mass Transfer, 90, 166 (2015).

[171] M. Jasinsky, E. Majhrzak, L. Turchan. Appl. Math. Model., 40, 750 (2016).

[172] J. Zhou, Y. Zhang, J.K. Chen. Int. J. Thermal Sci., 48, 1477 (2009).

[173] M.J. Noroozi, S. Saedodin, D.D. Gangi. Alexandria Eng. J., 55, 1745 (2016).

[174] C. Li, J. Miao, K. Yang, X. Guo, J. Tu, P. Huang, D. Zhang. J. Appl. Phys., 123, 174906 (2018).

[175] P. Kumar, D. Kumar, K.N. Rai. J. Thermal Biol., 49-50, 98 (2015).

[176] J.R. Ho, C.P. Kuo, W.S. Jiaung. Int. J. Heat Mass Transfer, 46, 55 (2013).

[177] A. Moradi, H. Ahmadikia. J. Eng. Med., 226, 406 (2012).

[178] H. Ahmadikia, A. Moradi. Heat Mass Transfer, 48, 2559 (2012).

[179] K.C. Li, H.T. Chen. Int. J. Heat Mass Transfer, 52, 1185 (2009).

[180] V. Borjalilou, M. Asghari, E.J. Bagheri. Thermal Stresses, 42, 1 (2019).

[181] Y. Chou, R. Yang. J. Int. J. Heat Mass Transfer, 52, 239 (2009).

[182] S.C. Chang. J. Comput. Phys., 119, 295 (1995).

[183] C.Y. Loh, S.C. Hultgren, S.C. Chang. AIAA J., 39, 794 (2001).

[184] A.H. Akbarzadeh, Y.Y. Cui, Z.T. Chen. RSC Adv., 7, 13623 (2017).

[185] A. Green, P. Naghdi. Proc. Roy. Soc. L, 357, 253 (1991).

[186] A. Green, P. Naghdi. J. Thermal Stresses, 15, 253 (1992).

[187] S.K.R. Choudhuri. J. Thermal Sci., 30, 231 (2016).

[188] A.H. Akbarzadeh, J. Fu, Z. Chen. Trans. Canadian Soc. Mech. Eng., 38, 155 (2014).

[189] R. Kumar, A.K. Vashishth, S. Ghangas. Int. J. Appl. Mech. Eng., 24, 603 (2019). 
[190] R. Kumar, V. Gupta. Mech. Adv. Mater. Struct., 23, 896 (2016).

[191] R.A. Guyer, J.A. Krumhansl. Phys. Rev., 148, 766 (1966).

[192] R.A. Guyer, J.A. Krumhansl. Phys. Rev., 148, 778 (1966).

[193] M. Calvo-Schwartzwalder, T.G. Meyers, M.G. Hennessy. arXiv: 19055.06320 [cond-mat.mes-hall], (2019).

[194] G. Chen. Phys. Rev. Lett., 86, 2297 (2001).

[195] G. Chen. J. Heat Transfer, 124, 320 (2002).

[196] H.L. Li, B.Y. Cao. Nanosc. Microsc. Thermophys. Eng., 23, 10 (2018).

[197] R. Yang, G. Chen, M. Laroche, Y. Taur. Trans. ASME, 127, 298 (2005).

[198] P.B. Allen. Phys. Rev. B, 97, 134307 (2018).

[199] C. Hua, L. Lindsay, X. Chen, A.J. Minnich. arXiv: 1902.10020 [cond-mat.mtrl-sci], (2019).

[200] Y. Guo, M. Wang. Phys. Rev. B, 97, 035421 (2018).

[201] A. Cepellotti, N. Marzari. Phys. Rev. X, 6, 041013 (2016).

[202] R.C. Tolman. Phys. Rev., 35, 904 (1930).

[203] M. Wang, N. Yang, Z.Y. Guo. J. Appl. Phys., 110, 064310 (2011).

[204] Z.Y. Guo. J. Eng. Thermophys., 27, 631 (2006).

[205] Y. Dong, B.Y. Cao, Z.Y. Guo. Phys. Rev. E, 87, 032150 (2013).

[206] H.D. Wang.Theoretical, Experimental Studies on NonFourier Heat Conduction Based on Thermomass Theory. (Springer, 2014).

[207] Y. Dong, B.Y. Cao, Z.Y. Guo. J. Appl. Phys., 110, 063504 (2011).

[208] M. Wang, B.Y. Cao, Z.Y. Guo. Front. Heat Mass Transfer, 1, 013004 (2010).

[209] J. Wu, Z. Guo, B. Song. Tsinghua Sci. Technol., 14, 12 (2009).

[210] Y. Dong, B.Y. Cao, Z.Y. Guo. Phys. Rev. E, 85, 061107 (2012).

[211] С.Л. Соболев. УФН, 167, 1045 (1997).

[212] P. Van, T. Fulop. Ann. Phys., 524, 470 (2012).

[213] R. Kovacs, P. Van. Int. J. Heat Mass Transfer, 83, 613 (2015).

[214] A. Sellitto, V.A. Cimmelli. J. Heat Transfer, 134 (2012).

[215] G.J. Lebon. Non-Equilib. Thermodyn., 39, 35 (2014).

[216] D. Jou, V.A. Cimmelli. Commun. Appl. Industr. Math., 7, 196 (2016).

[217] A. Sellitto, P. Rogolino, I. Carlomagno. Commun. Appl. Industr. Math., 7, 39 (2016).

[218] P. Rogolino, R. Kovacs, P. Van, V.A. Cimmelli. arXiv: 1709.05502 [cond-mat,stat-mech], (2018).

[219] С.Г. Самко, А.А. Килбас, О.И. Маричев. Интегралы u производные дробного порядка и некоторые их приложсения. (Наука и техника, Минск, 1987).

[220] А.М. Нахушев. Дробное исчисление и его применение. (Физматлит, Москва, 2003).

[221] В.А. Нахушева. Дифференциальные уравнения математических моделей нелокальных процессов. (Наука, M., 2006)

[222] В.Е. Тарасов. Модели теоретической физики с интегро-дибберенцированием дробного порядка. (Higher Education Press, Москва-Ижевск, 2011).

[223] F. Mainardi, R. Goremo. Int. J. Theor. Appl., 10, 269 (2007).

[224] M. Delkhosh. Appl. Math. Phys., 1, 103 (2013).

[225] I. Podlubny. Fractional Differential Equations. (AP, 1998).

[226] C. Li, D. Qian, Y.Q. Chen. Discr. Dyn. Nat. Soc., 2011, 562493 (2011).
[227] K.B. Oldham, J. Spanier. The Fractional Calculus. (AP, 1974).

[228] V.E. Tarasov. Int. J. Heat Mass Transfer, 93, 427430 (2016).

[229] A.A. Kilbas, H.M. Srivastave, J.J. Trujillo. Theory, Applications of Fractional Differential Equations. (North Holland, 2006).

[230] B. Li, J. Wang. Phys. Rev. Lett., 91, 044301 (2003).

[231] Y. Yu, D. Xu, Y.S. Xu, Q. Zhang. Appl. Math. Model., 40, 23 (2016).

[232] M. Rahimy. Appl. Math. Sci., 4, 2453 (2010).

[233] S.X. Deng, X.X. Ge. Thermal Sci., 23, 1671 (2019).

[234] J.H. He, F. Liu. Nonlinear Sci. Lett., 4, 15 (2013).

[235] V.D. Beybalaev. Матем. Модел., 21, 55 (2009).

[236] V.D. Beybalaev, A.A. Aliverdiev, R.A. Magomedov, R.R. Meilanov, E.N. Akhmedov. Vestn. Samar. Gos. Techn. Univ., Ser. Fiz.-Mat. Nauki, 21, 376387 (2017).

[237] J.H. He, Z.B. Li, Q.J. Wang. King Saud Univ. — Sci., 28, 190 (2016).

[238] Q.L. Wang, J.H. He, Z.B. Li. Thermal Sci., 16, 339 (2012).

[239] Р.П. Мейланов, М.Р. Шабанова. ЖТФ, 81, 1 (2011).

[240] V.R. Voller, F. Falcini, R. Garcia. Phys. Rev, E, 87, 042401 (2013).

[241] R. Meylanov, M. Shabanova, E. Akhmedov. Int. Rev. Chem. Eng., 3, 810813 (2011).

[242] D. Sierociuk, A. Dzielinski, G. Sarwas, I. Petr, I. Podlubny, T. Skovranek. Phil. Trans., Series A, 371, 20120146 (2013).

[243] R.S. Damor, S. Kumar, A.K. Shukla. J. Mech. Med. Biology, 14, 1450018 (2014).

[244] R.S. Damor, S. Kumar, A.K. Shukla. Am. J. Math. Analysis, 1, 20 (2013).

[245] R.S. Damor, S. Kumar, A.K. Shukla. Fractional Differential Calculus, 5, 43 (2015)

[246] M. Ezzat, N. Al-Sowayan, Z. Al-Muhiameed, S. Ezzat. Heat Mass Transfer, 50, 907914 (2014).

[247] L.L. Ferras, N.J. Ford, M.L. Morgado, J.M. Nobrea, M.S. Rebelo. Fract. Calculus Appl. Anal., 18, 1080 (2015).

[248] J. Singh, P.K. Gupta, K.N. Rai. Math. Comput. Model., 54, 23162325 (2011).

[249] M. Zingales. Comm. Nonlin. Num. Simul., 19, 3938 (2014).

[250] M. Zingales. Int. J. Heat Mass Transfer, 67, 593 (2013).

[251] N.J. Burch, R.B. Lehoucq. SAND2010-8783P, (2010).

[252] L. Liu, L.C. Zheng, F.W. Liu, X.X. Zhang. Int. J. Heat Mass Transfer, 103, 1191 (2016).

[253] B. Yu, X. Jiang, C. Wan. Appl. Math. Comp., 274, 106 (2016).

[254] P. Kumar, D. Kumar, K.N. Rai. Proc. Eng., 127, 56 (2015).

[255] X. Jiang, H. Qi. J. Phys. A: Math. Theor., 45, 485101 (2012).

[256] H.T. Qi, H.Y. Xu, X.W. Guo. Appl. Math. Comput., 186, 286 (2007).

[257] T.N. Mishra, K.N. Rai. Propuls. Power Res., 5 (1), 45 (2016).

[258] Л.И. Мороз, А.Г. Масловская. Матем. матем. модел., 2, 29 (2019).

[259] J. Christov. Thermal Sci., 20, 757 (2016).

[260] M. Caputo, M. Fabrizio. Progr. Fract. Di er. Appl. 1, 73 (2015).

[261] B.S. Alkahtani, A. Atangana. Thermal Sci., 21, 1 (2017).

[262] X.L. Yang, H.M. Srivastava, J.A.T. Machado. arXiv: 1601.01623, (2015).

[263] X.J. Yang, Y. Han, J. Li, W.X. Liu. Thermal Sci., 20, S717 (2016).

[264] C.C. Ji, W. Dai, Z.Z. Sun. J. Sci. Comput., 75, 1307 (2018).

[265] C.C. Ji, W. Dai, Z.Z. Sun. J. Sci. Comput., 81, 1767 (2019). 
[266] H.Y. Xu, X.Y. Jiang. Chin. Phys. B, 24, 034401 (2015).

[267] L. Liu, L. Zheng, F. Liu. Int. J. Heat Mass Transfer, 127, 165 (2018).

[268] M.A. Ezzat, A.S. El Karamany, M.A. Fayik. Arch. Appl. Math., 82, 557 (2012).

[269] M.A. Ezzat, A.A. El-Bary, M.A. Fayik. Mech. Adv. Mater. Struct., 20, 593 (2013).

[270] J. Fan, L.J. Wang. Appl. Phys., 109, 104202 (2011).

[271] D. Sarkar, A. Haji-Sheikh, A. Jain. Int. J. Heat Mass Transfer, 91, 602 (2015).

[272] A. Barletta, E. Zanchini. Heat mass transfer, 31, 443 (1996).

[273] S. Saerdodin, M. Torabi, H. Eskandar, M.J. Akbari. Comput. Anal. Appl., 13, 411 (2011).

[274] H. Ahmadikia, R. Fazlali, A. Moradi. Int. Commun. Heat Mass Transfer, 39, 121 (2012).

[275] H. Ahmadikia, A. Moradi, R. Fazlali, A. Basiri Parsa. J. Mech. Sci. Technol., 26, 1937 (2012).

[276] B. Kundu, D. Dewanjee. Case Stud. Therm. Eng., 5, 79 (2015).

[277] R.T. Al-Khairy, Z.M. Al-Ofey. J. Appl. Math., 2009, 504 (2009).

[278] H. Askarizadeh, H. Ahmadikia. Int. J. Engin., 27, 971 (2014).

[279] H. Askarizadeh, H. Ahmadikia. Heat Mass Transfer, 50, 1673 (2014).

[280] S.M. Lin. J. Mech. Med. Biol., 13, 1350063 (2013).

[281] R. Alkhairy. Appl. Math., 3, 1170 (2012).

[282] W. Dai, R. Nassar. Int. J. Heat Mass Transfer, 45, 1585 (2002).

[283] K. Zhukovski. Entropy, 19, 440 (2017).

[284] K. Zhukovski, D. Oskolkov, N. Gubina. Axioms, 7, 48 (2018).

[285] R. Kovacs. arXiv: 1804.05225 [cond-mat.sta.-mech], (2018).

[286] A. Getinkaya, O. Kiymaz. Math. Comput. Model., 57, 2349 (2013).

[287] Y. Povstenko. Cent. Eur. J. Phys., 11, 1284 (2013).

[288] Y. Povstenko. Comm. Appl. Industr. Math., 6, 1 (2014).

[289] Y. Povstenko. Entropy, 15, 4122 (2013).

[290] L. Junyi, X. Mingyu. J. Math. Anal. Appl., 351, 536 (2009).

[291] A.M. Yang, C. Cattani, H. Jafari, X. Yang. J. Abstract Appl. Anal., 2013, 462535 (2013).

[292] M. Kazemi, G.H. Erjaee. Iran. J. Sci. Tech., A3, 185 (2011).

[293] U. Ghosh, S. Sengupta, S. Sarkar, S. Das. Am. J. Math. Anal., 3, 32 (2015). 